\title{
A force-balance model for centrosome positioning and spindle elongation during Interphase and Anaphase B
}

\author{
Arittri Mallick, ${ }^{1, *}$ Apurba Sarkar,,${ }^{1, \dagger}$ and Raja Paul ${ }^{1, \ddagger}$ \\ ${ }^{1}$ Indian Association for the Cultivation of Science, Kolkata-700032, India
}

\begin{abstract}
A computational model in one dimension is proposed to position a single centrosome using astral microtubules (MTs) interacting with the cell cortex. The mechanism exploits mutually antagonistic pulling and pushing forces arising from the astral MTs' binding to cortical dynein motors in the actin-rich cell cortex and their buckling while growing against the cell cortex, respectively. The underlying mechanism of astral MTs is also extended to account for the elongation and positioning of the bipolar spindle during mitotic anaphase B. The model for bipolar spindle involves both IPMTs and astral MTs, can predict its elongation and positioning under various circumstances. The model reveals that the bipolar spindle elongation, weakened by decreasing overlap between the antiparallel interpolar microtubules (IPMTs) in the spindle mid-zone, is recovered by the astral MTs.
\end{abstract}

\section{INTRODUCTION}

Microtubules (MTs) form the basis of cellular architecture and dynamics that give rise to complex phenomena with significant implications in cell biology. The MTs can emanate from various cellular machinery and are very useful mechanical propellers of various cellular processes [1-7]. They interact with associated cellular components like molecular motors: both uni-polar and bipolar; the cell cortex, and even other MTs [8-10]. MTs are known to be actively involved in mechanical interactions that are crucial for critical cellular processes like centrosome positioning at the cell center during interphase $[2,11-17]$, bipolar spindle elongation and positioning during mitotic anaphase B [18-20] etc. Given that MTs play the chief mediator in these processes, it is essential to develop a computational model for studying these processes in great detail.

The centrosome is actively positioned at the cell center during the Interphase of the cell cycle $[2,11,12,16]$. It is subjected to a force field of pulling and pushing forces that are mediated by cortical motors and centrosome nucleated microtubules, respectively $[2,11,12]$. The centrosome, which is thought to be the primary microtubuleorganizing center(MTOC) in mammalian cells, can give rise to microtubules that grow isotropically toward the cell cortex [8]. These microtubules are called astral microtubules. Their dynamic instability parameters ensure the growth till the cell cortex [21] where their interaction with the cortical wall acts as the source of various force generating mechanisms. The astral MTs, on reaching the cell cortex, can associate with the cortical dynein motors that are already bound to the actin-rich cortical layer. Association of the astral MTs with the dynein motor is favored as these motors are minus-end directed. Minusend directed motion of the motors generate pulling force

\footnotetext{
*Electronic address: mallickarittri13@gmail.com

$\dagger$ Electronic address: apurbaphysics391@gmail.com

${ }^{\ddagger}$ Electronic address: raja.paul@iacs.res.in
}

(toward the cell cortex) on the centrosome through the astral MTs [11]. The event of buckling of MTs against a rigid barrier, when they are grown from a movable source, has long been established [22] and is known to generate a net push on the source, directed away from the rigid barrier. The astral MTs have been found to buckle against cell organelles and cortex to generate pushing force on the centrosome [11]. Eventually, a balance between these forces causes the centrosome to position itself at the cell center. During Interphase, fission yeast (Schizosaccharomyces pombe) employs a similar mechanism to retain the nuclei at the cell center, ensuring that the metaphase plate forms close to it [23]. S. pombe is an elongated cell with antiparallel bundles of microtubules arranged along the cell's long axis [23, 24]. Their minus ends appear to cluster on the nuclear envelope at places near its north and south poles [23]. The plus ends of these microtubules grow parallel to the long axis and bend against the membrane as they try to grow against the cell tips, producing transitory pushing force that eventually positions the nucleus at the cell's center [23].

The centrosome gets duplicated before the onset of mitosis [25, 26], aiming to build a bipolar array of microtubule-based machinery, called the mitotic spindle, which forms during metaphase [27, 28]. The metaphase spindle must maintain a stable distance between the poles with chromosomes in the spindle mid-zone while maintaining an equal distance from each pole via dynamic microtubules. The astral MTs between the spindle pole and the cortex interact with the cell cortex through dynein motors and directly, inducing microtubule buckling. The dynein motors tend to elongate the spindle while the buckling force tries to compress it [9]. The metaphase spindle interzone hosts KMTs in connection with the kinetochores. The junction between them is occupied by minus end-directed motors (such as dynein), which in unison with KMT plus end depolymerization give rise to a contraction in the spindle [29]. The spindle contraction is countered by another set of proteins known as 'chromokinesins' operating between the KMT tips and the surrounding chromosomal arms. These exert pushing force on the spindle poles [30] tending to move them 
apart. The compressive and extensile forces compete to maintain the stable spindle configuration [9].

Spindle elongation occurs during anaphase and is one of the major events that characterize a successful mitotic division. It is the manifestation of increasing distance between the spindle poles that constitute the bipolar spindle. The anaphase onset triggers the breakage of cohesin spring at the KT-KT junction of sister chromatids, disrupting the inter-polar attraction force. This disturbs the balance of forces, allowing the segregation of duplicated chromosomes and the subsequent elongation and positioning of the spindle. Numerous experimental studies suggest that it is crucial for successfully completing mitosis and forming healthy daughter cells. The segregation of duplicate chromosomes during anaphase is assisted [31, 32] by kinetochore-associated microtubule (KMT) dynamics (anaphase A) as well as elongation of the spindle (anaphase B). Thus, proper spindle elongation plays a role in ensuring that there is no chromosomal anomaly when the daughter cells are formed. It has been reported that in the blastomeres of $C$. elegans, the chromosome segregation takes place solely due to spindle elongation [33]. Timely elongation of the spindle positions the segregated chromosomes and centrosomes away from the center of the cell, determining the position of cytoplasmic cleavage during cytokinesis [8]. In this way, proper spindle elongation and positioning also facilitate cells to divide desired proportions of cytoplasm between the daughter cells.

Both the metaphase and anaphase spindles are found to be mechanically robust against any external perturbations. In a recent experimental study conducted on C. elegans cells [34], optical tweezers were employed to displace one of the poles in a bipolar spindle, and the impact of this displacement was investigated upon release. The displaced pole came back to its original position, indicating that the spindle behaves like a spring. The repositioning of the spindle at the cell center was due to the dynamics of the astral microtubules generating strong pushing force on the poles. The stiffness of the spring-like force generator increases during anaphase and precisely position the spindle suppressing the thermal fluctuations $[9,34]$.

The mechanism involved in spindle elongation has been studied extensively in the past decades. Experimental studies on Drosophila embryos [35] revealed that spindle elongation involves non-KMTs present in the spindle inter-zone, called interpolar microtubules (IPMTs), which grow from one pole and interact with the IPMTs growing from the other pole in the spindle interzone. The antiparallel IPMTs (originating from different poles) have a finite overlap where they are cross-linked by mitotic motors like bipolar kinesin-5 [36, 37]. These motors can generate sliding motion on the IPMTs that in turn get exerted on the poles, which are thus slid apart [35]. Each IPMT has a free dynamic end in the spindle interzone and a bound and much less dynamic end at the respective pole. The free MT end is designated the 'plus end' while the less dynamic end is designated the 'minus end'. Prior to the onset of anaphase B, the IPMTs exhibit a net polymerization [35] at their plus ends, and a net depolymerization at their minus ends. Because of this simultaneous polymerization and depolymerization of the IPMT plus ends and minus ends, respectively, the tubulin dimers that the IPMTs are composed of exhibit a net motion toward the respective poles. This rate of movement of the tubulin dimers along the length of the IPMT is referred to as IPMT flux rate [35]. The sliding action of the kinesin- 5 motors on the IPMTs is antagonized by this IPMT flux rate of the tubulin dimers, preventing the spindle from elongating [35] under their sliding force. The mechanism ensures a constant interpolar separation. The onset of anaphase $\mathrm{B}$ reduces the rate of depolymerization at the IPMT minus ends, causing a decrease in the rate of IPMT flux [35, 38]. This reduces its antagonizing influence causing the action of kinesin- 5 motors to become more effective in sliding the poles apart [35], thus resulting in the elongation of the spindle. Although this mechanism suffices in Drosophila embryos, it is not the general mechanism for spindle elongation. Experimental studies [39] have revealed that in most vertebrates, the astral MTs take part in spindle elongation. As stated earlier, the astral MTs emanate from each pole to grow and reach the cell cortex. The blastomeres of $C$. elegans showed marked dependence on these astral MTs for the elongation of the spindle [33] with the spindle interzone limiting the extent of this elongation. Most animals and protozoans depend on a secondary mechanism of spindle elongation [8] comprising of astral MTs. A study [18] in 2007 found that in the blastomeres of $C$. elegans, the kinesin-5 motor in the spindle midzone functions as a 'brake' on spindle elongation that resists the spindle's extension in the face of a fast pole-separating mechanism. In such scenarios, the spindle elongation is assisted by the astral MTs [40].

Therefore, the IPMT mediated mechanism is limited by the IPMT overlap and needs a secondary mechanism to elongate the spindle. The astral MTs are found [32] to interact with the actin-rich cell cortex on the inner side of the plasma membrane to generate a motor-mediated pulling force on the respective poles, much like the way pulling forces are generated on the centrosome during Interphase. Astral MTs also buckle at the cell cortex [21], due to their growth against the cortical barrier. The growth generates a push on the poles directed away from the cell cortex toward the cell center. Therefore, besides the IPMT sliding force, the mechanism involves an interplay between the pulling and pushing forces [32] that ultimately translates into spindle elongation and its positioning, much like the way a single centrosome positioning occurs during Interphase.

The overlap in single centrosomal positioning and bipolar spindle elongation mechanisms has been utilized to craft a one-dimensional computational model that employs astral MTs and their interactions with the cell cortex. The single centrosomal dynamics employs the 
growth of the astral MTs from both sides of the centrosome, making their interactions the major force component. The bipolar spindle comprising of two individual poles employs similar tactics, but only on one side, between each pole and the proximal cell cortex. The MTs growing from each pole into the spindle interzone become the IPMTs and contribute to the sliding apart of the individual poles.

While the model for the IPMT-mediated spindle elongation has been studied earlier, it is limited by the overlap-dependent elongation [35]. As the poles separate, the IPMT overlap falls, and the sliding motors operate with a sliding velocity close to their stall velocity, which is not sufficient to attain the desired elongation or positioning of the spindle. Experimental studies [18, 40] indicate that in such circumstances, the astral MTs assist in spindle elongation. Although the mechanism is qualitatively explained, to our knowledge, a computational compilation of the same has not yet taken precedence. Here, we explore and develop upon an earlier model [35] for the IPMT-mediated elongation of the spindle and augment it with the astral MT mechanism as stated above.

While investigating the single centrosomal positioning, we exploit the basic model in the framework of a bipolar spindle for studying elongation kinetics of the latter. The models are implemented to explore the acceptable range of parameters that facilitate the desired patterns for single centrosome and bipolar spindles. The onedimensional model can confer the duration of anaphase $\mathrm{B}$ and allow its application on rod-shaped cells like $C$. elegans [41] to a close approximation. The model is further employed to study the impact of cell diameter, average MT length, the effect of MT buckling, and dynein-related force parameters on the centrosomal and bipolar spindle dynamics revealing exciting insights into their roles and applications.

\section{MODEL}

\section{A. Positioning of a Centrosome}

We propose a model for centrosome positioning in one dimension. The forces acting on the centrosome (henceforth denoted by CS) in interphase cells are primarily mediated by astral MTs that grow to the cell cortex and interact with it. The minus-end directed cortical dynein motors bind to the astral MTs, causing them to exert a force on the CS toward the cell cortex. Again, astral MTs' growth against the cell cortex causes them to buckle and apply a pushing force on the CS, directed away from the cell cortex. We assume that the astral MTs, modeled as polymeric rods, undergo first-order Euler buckling while growing against the cortex [22] generating a pushing force on the CS, which is inversely proportional to the square of the MT length. Thus, shorter MTs that reach the cell cortex generate a higher push on the CS. The dynein motors are considered to be uni- formly distributed on the actin-rich cell cortex and quantified by a linear density. The number of dynein motors bound to a given astral MT in the cell cortex depends on the extent of the MT that slides along the cortex. A steric repulsion from the cell periphery confines the CS within the cell cytoplasm whenever an overlap is set to occur between them.

Fig. 1 shows the schematic of the mechanism of force balance. The region of interest on the cellular axis is identical to the cell length, which henceforth is referred to as the cell diameter and denoted by $d_{\text {cell }}$. Thus, the cellular axis along the $X$-direction is bounded by the cell boundaries at $x=0$ and $x=d_{c e l l}$. The instantaneous position of the CS is denoted by $x_{\text {cent }}(t)$. The astral MTs undergo dynamic instability [42], due to stochastic de-polymerization and polymerization which is modelled by four parameters: rescue frequency $f_{\text {res }}$, catastrophe frequency $f_{\text {cat }}$, growth velocity $v_{g}$ and shrinkage velocity $v_{s}$ [43]. The average MT length corresponding to the dynamic instability parameters is given by $[43,44]$ :

$$
L_{\text {avg }}=\frac{v_{g} v_{s}}{f_{\text {cat }} v_{s}-f_{\text {res }} v_{g}}
$$

The amount of pushing force produced by an astral MT of unit length is defined by a buckling amplitude $A_{\text {buck }}$ which appears to be about ten times the flexural rigidity of the astral MTs [11]. The linear density of dynein motors on the cortex is denoted by $k_{d y n}$ and is uniform in character. Each motor engaged with an astral MT applies a mean force characterized by $F_{d y n}$. MTs grow as per the dynamic instability parameters on both sides of the CS toward the cell cortex. The position of the cortex is denoted by $x_{c o r}$ and the tip of a given MT as $x_{\text {tip }}$.

The growth of the astral MTs against the cell cortex modifies the growth velocity [22] and the catastrophe frequency [45] causing the former to decrease and the latter to increase with progressing growth against the cell cortex. The growth against the cell cortex is not favored as it generates a pushing force opposite to the direction of growth of the MTs. The astral MTs exhibit resistance to this growth by modifying the dynamic instability parameters $v_{g}$ and $f_{\text {cat }}$ based on the pushing force experienced by them [12] as shown.

$$
\begin{gathered}
v_{g}^{\text {modified }}=v_{g} \exp \frac{-f_{\text {push }}}{F_{\text {stall }}} \\
f_{\text {cat }}^{\text {modified }}=\frac{f_{\text {cat }}^{\text {stall }}}{1+\left(\frac{f_{\text {cat }}^{\text {stall }}}{f_{\text {cat }}}-1\right) \exp \frac{-f_{\text {push }}}{F_{\text {stall }}}}
\end{gathered}
$$

Here, $F_{\text {stall }}$ refers to the load force on astral MTs that causes them to stall as they grow against the cell cortex; $f_{\text {cat }}^{\text {stall }}$ is the catastrophe frequency of a stalled astral MT [45] and $f_{\text {push }}$ is the net load force that opposes MT 

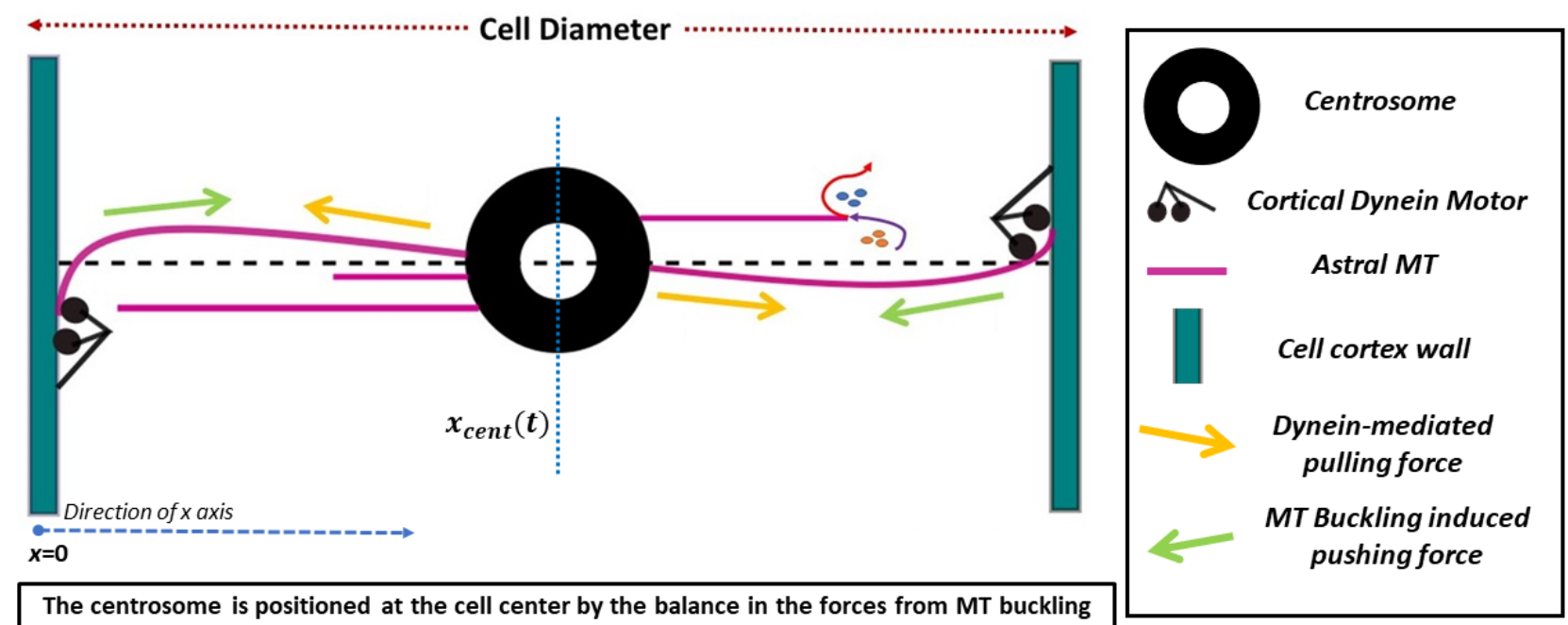

The centrosome is positioned at the cell center by the balance in the forces from
and cortical dynein originating in the cell cortex on both sides.

FIG. 1: One-dimensional model for the positioning of a centrosome in a cell. The astral MTs undergo first-order Euler buckling upon growth against the cell cortex. The astral MTs exhibit dynamic instability indicated by depolymerization and polymerization of the MT tips. The arrows indicate the direction of the respective forces. The direction of the $X$-axis is shown in the picture. The horizontal dotted line, on which the centrosome rests, signifies the cellular axis along which it is restricted to move.

growth against the cortex at a given instant. The modifications of the instability parameters stated in Eqs. (2) and (3) are applicable only after the astral MTs have reached the cortex. At the cortex, the MTs experience pulling and pushing forces. Let the pull experienced by the $i^{t h}$ astral MT emanating from the CS be $f_{\text {pull }}^{i}$ and the push be $f_{\text {push }}^{i}$. If $L_{M T}^{i}$ is the length of the $\mathrm{i}^{\mathrm{th}}$ astral MT, we can write:

$$
\begin{gathered}
f_{\text {pull }}^{i}=k_{d y n} F_{d y n}\left[L_{M T}^{i}-\left|\left(x_{c o r}-x_{c e n t}\right)\right|\right] \\
f_{\text {push }}^{i}=\frac{A_{b u c k}}{L_{M T}^{i}{ }^{2}} ; L_{M T}^{i}=x_{t i p}^{i}-x_{c e n t}
\end{gathered}
$$

The length of an astral MT is measured from the position of its origin at the CS to its tip denoted by $x_{t i p}$. Subtracting the separation between the CS and the cortex from the MT length (see Eq. (4)), we obtain the length of the MT sliding along the cell cortex. The dynein motors can bind to this length and contribute to pulling force. Summing over the superscript ' $\mathrm{i}$ ' in Eqs. (4)and (5) would give the total pulling and pushing forces, respectively.

Now, suppose the CS moves toward the cortex with an instantaneous velocity. In that case, it experiences a drag force against its motion in the cell cytoplasm proportional to this velocity due to the drag coefficient of the cytoplasm, denoted by $\mu$. Thus, the force balance equation for the CS becomes:

$$
\begin{aligned}
\mu \frac{d x_{\text {cent }}}{d t}= & \sum_{i} f_{\text {pull }}^{i}{ }^{\text {right }}-\sum_{j} f_{\text {pull }}^{j}{ }^{\text {left }}-\sum_{i} f_{\text {push }}^{i}{ }^{\text {right }} \\
& +\sum_{j} f_{\text {push }}^{j}{ }^{\text {left }}-f_{\text {steric }}^{\text {cor }}{ }^{\text {right }}+f_{\text {steric }}^{\text {cor }} \text { left }^{\text {la }}
\end{aligned}
$$

The superscripts 'i' and 'j' denote the astral MTs interacting with the right and left cortex, respectively. The steric repulsion force, denoted by $f_{\text {steric }}^{c o r}$ acts between the CS and the cell cortex when they almost overlap with each other and is inverse squared in nature. The cell cortex is separated from the cell boundary at $x=0$ and $x=d_{\text {cell }}$ by a distance of $0.75 \mu \mathrm{m}$. The steric force is quantified by:

$$
f_{\text {steric }}^{c o r}=\frac{A_{\text {steric }}}{\left[x_{\text {cent }}-x_{c o r}\right]^{2}}
$$

The amplitude of steric repulsion force is denoted by $A_{\text {steric }}$. The buckling-mediated pushing and the dyneinmediated pulling forces apply only after the astral MTs have reached the cell cortex. Likewise, the steric force contributes to the repulsion between the cortex and the CS only after the CS has reached within a distance of $1.5 \mu \mathrm{m}$ from the cell cortex. We solve eq. 6 computationally to evaluate $x_{\text {cent }}(t)$ to obtain the stochastic dynamics of CS.

\section{B. Bipolar Spindle Elongation during Anaphase B}

As shown in Fig. 2, the CSs or the individual poles in a bipolar spindle experience similar forces as discussed 


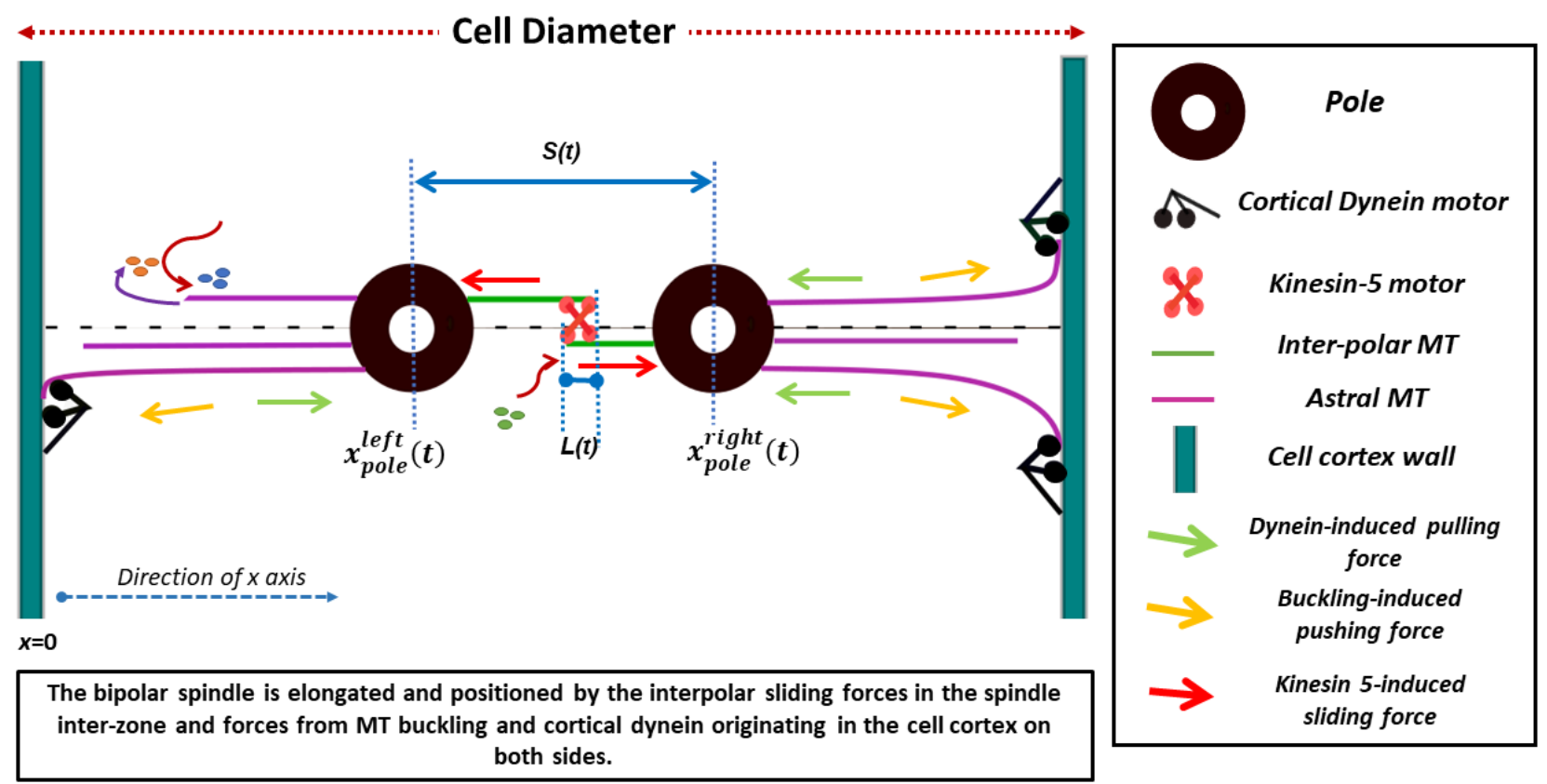

FIG. 2: One-dimensional model for the elongation of bipolar spindle during Anaphase B. Here, the force-generating mechanisms mediated by the astral MTs on individual poles are similar to the single CS scenario as in Fig. 1. Antiparallel arrays of IPMTs crosslink in the spindle interzone via bipolar kinesin-5 which slide the IPMTs apart.

previously for the single CS, except in the spindle interzone. To describe forces in this region, we exploit the previous model of IPMT mediated spindle elongation in Drosophila embryo [35]. The spindle interzone consists of IPMTs that interact in antiparallel pairs (MTs emanating from opposite poles). Overlapping IPMTs get crosslinked by plus-end directed kinesin- 5 that slide the MTs apart as shown in Fig. 2. The IPMTs undergo depolymerization at their minus ends and net polymerization at their plus ends [35], the rates of which are denoted by $V_{d}^{-}$and $V_{p}^{+}$respectively. The simultaneous depolymerization and polymerization of the IPMTs give rise to MT flux via the tubulin dimers that antagonize the sliding action of kinesin-5 [35]. The onset of anaphase B triggers a decrease in the rate of IPMT depolymerization at the poles [35] causing the IPMT sliding to take effect. The kinesin-5 motor follows a linear force-velocity relation as shown below:

$$
F_{k i n}=F_{m a x}^{\text {stall }}\left(1-\frac{V_{k i n}}{V_{\text {max }}^{\text {unload }}}\right)
$$

The kinesin- 5 motor moves with a velocity $V_{k i n}$ under the load of $F_{k i n} . F_{\text {max }}^{\text {stall }}$ refers to the load under which the motor stalls causing the motor velocity $V_{k i n}$ to reduce to zero. Conversely, $V_{\max }^{\text {unload }}$ refers to the maximum unloaded velocity of the motor, that is, when the load force $F_{k i n}$ on it becomes zero.

The velocity of kinesin- 5 equals the velocity with which the IPMTs are slid apart. Let the velocity be given by $V_{\text {sliding. }}$ The dynamic antiparallel IPMT overlap denoted by $L(t)$, is shown in Fig. 2. Thus, we have [35]:

$$
\frac{d L(t)}{d t}=2\left(V_{p}^{+}-V_{\text {sliding }}\right)
$$

The factor 2 in the equation accounts for the assumed symmetry of the spindle interzone. Eq. (9) shows that the rate of overlap increases with increasing rates of IPMT plus-end polymerization and decreases as the rate of sliding of the antiparallel IPMTs increases. Now, let the instantaneous position of any one pole be denoted by $x_{\text {pole }}$. Since the flux due to de-polymerization at the IPMT minus-ends opposes the pole separation, we have [35]:

$$
\frac{d x_{\text {pole }}}{d t}=V_{\text {sliding }}-V_{d}^{-}
$$

Factor 2 does not appear here because the above equation accounts for only one pole. The velocity of the pole's movement, in Eq. (10), is favored by the IPMT sliding rates and is antagonized by increasing rates of tubulin flux along the IPMTs facilitated by the rate of IPMT minus-end depolymerization. As this pole moves through the cytoplasm toward the cortex, it experiences a drag force due to its drag coefficient $\mu$, proportional to its instantaneous velocity. The resistance is countered by the action of the sliding motors in the spindle interzone. The number of kinesin-5 motors engaged between any set of antiparallel IPMTs is represented by a linear density $k_{k i n}$ and is proportional to the corresponding IPMT overlap. If $N$ implies the total number of IPMT arrays in the spindle interzone [35], the force balance equation for any one pole is: 


$$
\mu \frac{d x_{p o l e}}{d t}=k_{k i n} N L(t) F_{k i n}
$$

By algebraic manipulation of Eqs. (9), (10), and (11), we finally arrive at the following expression:

$$
V_{\text {sliding }}=\frac{\alpha L(t)}{1+\alpha L(t)} V_{\max }^{\text {unload }}+\frac{1}{1+\alpha L(t)} V_{d}^{-}
$$

where $\alpha=\frac{k_{\text {kin }} N F_{\text {max }}^{\text {stall }}}{\mu V_{\text {max }}^{\text {und }} \text { id }}$ in Eq. (12) is a constant and denotes the strength of the IPMT sliding force against the viscous drag offered by the cytoplasm. Note that(see 12), for a small IPMT overlap, the sliding velocity tends to converge to the rate of de-polymerization at the IPMT minus end. Since this rate decreases at the onset of anaphase $\mathrm{B}$, the elongation of the bipolar spindle is hindered [35]. Substituting Eq. (12) in Eq. (9) and (10), we obtain the IPMT contribution to the dynamics of any given pole:

$$
\frac{d L(t)}{d t}=2\left(V_{p}^{+}-\frac{\alpha L(t)}{1+\alpha L(t)} V_{\max }^{\text {unload }}-\frac{1}{1+\alpha L(t)} V_{d}^{-}\right)
$$

$$
\frac{d x_{\text {pole }}}{d t}=\frac{\alpha L(t)}{1+\alpha L(t)}\left(V_{\text {max }}^{\text {unload }}-V_{d}^{-}\right)
$$

Since the spindle elongation is hindered due to decreasing overlap between the antiparallel IPMTs, the astral MTs must assist in further elongation. The force characteristic of astral MTs in a spindle is similar to the single CS, and the dynamic instability parameters of astral MTs emanating from one pole are independent of the other. The pulling and the pushing forces are described by Eqs. (4) and (5), respectively, whereas, the altered dynamical parameters are expressed in Eq. (2), and (3). If the instantaneous position of the right and the left poles are denoted by $x_{\text {pole }}^{\text {right }}$ and $x_{\text {pole }}^{\text {left }}$ respectively, and length of the $\mathrm{i}^{\text {th }}$ astral MT is $L_{M T}^{i}$, then we have:

$$
\begin{aligned}
\frac{d x_{\text {pole }}^{\text {right }}}{d t} & =\frac{\alpha L(t)}{1+\alpha L(t)}\left(V_{\text {max }}^{\text {unload }}-V_{d}^{-}\right) \\
& +\left[\frac{\sum_{i} f_{\text {pull }}^{i}-\sum_{i} f_{\text {push }}^{i}-f_{\text {steric }}^{\text {cor }}+f_{\text {steric }}^{\text {interpolar }}}{\mu}\right]
\end{aligned}
$$

$$
\begin{aligned}
-\frac{d x_{\text {pole }}^{\text {left }}}{d t} & =\frac{\alpha L(t)}{1+\alpha L(t)}\left(V_{\text {max }}^{\text {unload }}-V_{d}^{-}\right) \\
+ & {\left[\frac{\sum_{j} f_{\text {pull }}^{j}-\sum_{j} f_{\text {push }}^{j}-f_{\text {steric }}^{\text {cor }}+f_{\text {steric }}^{\text {interpolar }}}{\mu}\right] }
\end{aligned}
$$

The '-' sign on the left-hand side of Eq. (16) accounts for the position $x_{\text {pole }}^{\text {left }}$ becoming smaller in magnitude as the spindle elongates and the left pole translates toward the left cortex. The superscripts ' $i$ ' and ' $j$ ' denote the astral MTs interacting with the right and left cortex respectively. The interpolar steric repulsion, denoted by $f_{\text {steric }}^{\text {interpolar }}$, is included to avoid the poles from crossing each other:

$$
f_{\text {steric }}^{\text {interpolar }}=\frac{A_{\text {steric }}^{\text {interpolar }}}{\left(x_{\text {pole }}^{\text {right }}-x_{\text {pole }}^{\text {left }}\right)^{2}}
$$

The amplitude of this steric force $A_{\text {steric }}^{\text {interpor }}$ is non-zero only when poles are within a particular 'cut-off' proximity $\sim 1 \mu \mathrm{m}$. The steric repulsion between the cortex and the respective poles (i.e., $f_{\text {steric }}^{\text {cor }}$ ) stays the same as in Eq. (7). Eqs. (13), (15) and (16) are simultaneously solved to obtain the interpolar separation $S(t)$ as:

$$
S(t)=x_{\text {pole }}^{\text {right }}(t)-x_{\text {pole }}^{\text {left }}(t)
$$

Thus, the model discussed here evaluates $S(t)$ as the bipolar spindle elongation during anaphase B in a timedependent manner.

\section{COMPUTATIONAL TECHNIQUES}

The MTs were assigned dynamic instability parameters (refer to Table I) to exhibit stochastic growth and shrinkage motion at their plus ends. In contrast, the minus end remained attached to the centrosomal poles. The probability of catastrophe and rescue during a single time step (taken as $0.1 \mathrm{~s}$ ) was given by,

$$
P_{\text {res } / \text { cat }}=f_{\text {res } / \text { cat }} * \text { time step }
$$

The choice of events relating to rescue and catastrophe of MTs was made by comparing the probabilities to a random number generated uniformly between 0 and 1 at each computational time step. Dynamic instability of the microtubules was evaluated at each time step. For the single CS positioning, simulations were carried out for different initial positions of the CS, varied in steps of $5 \mu \mathrm{m}$ along the cellular axis, including extreme and intermediate cellular zones. The astral MTs' tip positions at $t=0 \mathrm{~s}$ were randomly distributed between the CS and the cell cortex on both sides. The astral MTs grew according to their dynamic instability parameters (see Table I) to reach the cell cortex. The random rescue and catastrophe events at their plus end allowed them to interact with the cell cortex randomly. A counter was introduced to estimate the number of astral MTs reaching the cell cortex at each step. Monitoring the dynamics of each astral MT (via $x_{t i p}$ ) allowed us to calculate their length $L_{M T}$ (see 5). This data was used to evaluate the average dynein mediated pulling and MT buckling mediated pushing forces following Eqs. (4) and (5), acting 
bioRxiv preprint doi: https://doi.org/10.1101/2021.10.04.463000; this version posted October 4, 2021. The copyright holder for this preprint (which was not certified by peer review) is the author/funder, who has granted bioRxiv a license to display the preprint in perpetuity. It is made available under aCC-BY-NC-ND 4.0 International license.

\begin{tabular}{|c|c|c|c|c|}
\hline \multicolumn{5}{|c|}{ Table I: Parameters and their definition } \\
\hline Parameter Definition (unit) & Parameter Abbreviation & Value & Range studied & Reference \\
\hline Cell Radius $(\mu m)$ & $r_{\text {cell }}$ & 20 & $10-30$ & {$[11,41]$} \\
\hline Cell diameter $(\mu m)$ & $d_{\text {cell }}$ & 40 & $20-60$ & {$[11,41]$} \\
\hline Steric Amplitude $\left(p N-\mu m^{2}\right)$ & $A_{\text {steric }}$ & 150 & - & This study \\
\hline Interpolar steric amplitude $\left(p N-\mu m^{2}\right)$ & $A_{\text {steric }}^{\text {interpolar }}$ & 150 & - & This study \\
\hline $\begin{array}{l}\text { Astral MT stall force } \\
\qquad(p N)\end{array}$ & $F_{\text {stall }}$ & 1.3 & - & $\begin{array}{l}\text { This study, } \\
{[22,45,46]}\end{array}$ \\
\hline Astral MT average length $(\mu m)$ & $L_{\text {avg }}^{A M T}$ & 40 & $5-60$ & {$[11,45]$} \\
\hline Astral MT flexural rigidity $\left(p N-\mu m^{2}\right)$ & - & $\sim 18$ & - & [11] \\
\hline Astral MT buckling amplitude $\left(p N-\mu m^{2}\right)$ & $A_{\text {buck }}$ & 180 & - & {$[11,45]$} \\
\hline Number of astral MTs & - & 50 & - & [11] \\
\hline Number of IPMTs in spindle inter-zone & $\mathrm{N}$ & 30 & - & {$[35,47]$} \\
\hline Drag Coefficient $(p N-s e c / \mu m)$ & $\mu$ & 1000 & - & {$[48]$} \\
\hline Stall force for Kinesin-5 $((p N))$ & $F_{\max }^{\text {stall }}$ & 1 & - & {$[49]$} \\
\hline Unloaded Velocity of Kinesin-5 $(\mu \mathrm{m} / \mathrm{sec})$ & $V_{\text {max }}^{\text {unload }}$ & 0.07 & - & {$[35,50]$} \\
\hline Linear Density of Kinesin-5 on IPMT $(/ \mu m)$ & $k_{k i n}$ & 20 & - & {$[35,51]$} \\
\hline Force exerted by single cortical dynein motor $(p N)$ & $F_{d y n}$ & 1.25 & - & {$[52,53]$} \\
\hline Linear Density of cortical dynein motor $(/ \mu m)$ & $k_{d y n}$ & 5 & $1-5$ & {$[45,46]$} \\
\hline IPMT catastrophe frequency $\left(s^{-} 1\right)$ & $f_{\text {cat }}^{I P M T}$ & 0.16 & - & {$[35,54]$} \\
\hline IPMT rescue frequency $\left(s^{-} 1\right)$ & $f_{\text {res }}^{I P M T}$ & 0.2 & - & {$[35,54]$} \\
\hline IPMT growth velocity $(\mu \mathrm{m} / \mathrm{s})$ & $v_{g}^{I P M T}$ & 0.16 & - & {$[35,54]$} \\
\hline IPMT shrinkage velocity $(\mu \mathrm{m} / \mathrm{s})$ & $v_{s}^{I P M T}$ & 0.16 & - & {$[35,54]$} \\
\hline Astral MT catastrophe frequency $\left(s^{-} 1\right)$ & $f_{\text {cat }}^{A M T}$ & 0.033 & - & {$[2]$} \\
\hline Astral MT rescue frequency $\left(s^{-} 1\right)$ & $f_{\text {res }}^{A M T}$ & 0.067 & - & {$[2,12]$} \\
\hline Astral MT growth velocity $(\mu \mathrm{m} / \mathrm{s})$ & $v_{g}^{A M T}$ & 0.24 & $0.12-0.25$ & {$[53,55]$} \\
\hline Astral MT shrinkage velocity $(\mu \mathrm{m} / \mathrm{s})$ & $v_{s}^{A M T}$ & 0.6 & $0.59-0.8$ & {$[55]$} \\
\hline Stall frequency of astral MT $\left(s^{-} 1\right)$ & $f_{\text {cat }}^{\text {stall }}$ & 0.042 & - & {$[12,45]$} \\
\hline
\end{tabular}

on the CS at each time step. The pushing force so calculated was used to evaluate the modified growth velocity and the catastrophe frequency as per Eqs. (2) and (3). As indicated before, the MT growth on either side of the CS was dynamically independent. The counters measuring the number of astral MTs interacting with the cell cortex at any given time step were found to vary, implying fluctuations in their respective contributions to the resultant force. Using these data at each time step, Eq. (6) was solved to obtain the position of the CS. This process was repeated for 1000 ensembles to obtain the mean and the standard deviation in the data.

To study the bipolar spindle elongation, the spindle was symmetrically positioned with an initial interpolar separation of $10 \mu m$ (held constant in all the simulations). The overlap of the IPMTs was obtained at each time step from Eq. (13) and fed into Eqs. (15) and (16) for obtaining the dynamics of the two poles. The astral MTs were simulated similar to the single CS scenario. For IPMTs, only positive overlaps between the antiparallel arrays were considered. A negative overlap meant that the IPMTs were no longer overlapped. Thus, the sign of the overlap was checked at each time step. The stochastic dynamics of IPMTs allowed them to regain a non-zero overlap even if they had lost contact earlier.

The average polymerization rate $V_{p}^{+}$was found at each time step by monitoring the dynamic instability of the IPMTs and calculating their growth rate at each time step. $V_{p}^{+}$remained constant during each time step and was fed to Eq. (13) as a parameter. Also, since the depolymerization rate $V_{d}^{-}$of the IPMT minus ends considerably decreases at the onset of anaphase B [35, 38], it was considered to be zero for simplicity.

Using the expressions for the pulling and pushing forces from Eqs. (4) and (5), the forces were estimated for each pole. Based on the magnitude of the pushing force, dynamic instability parameters of the MTs were updated at each time step. Forces and IPMT overlap evaluated at each time step were used to solve the set of three differential Eqs (13), (15) and (16) simultaneously to obtain the interpolar separation as well as dynamics of individual poles. The process was repeated for 1000 ensembles. The numerical codes were implemented in Fortran, and the differential equations governing the system dynamics were solved numerically using Runge-Kutta methods [56]. 


\section{RESULTS}

\section{A. Single CS Dynamics}

\section{Buckling of astral MT plays a major role in positioning the CS at the cell center}

A single CS is considered in one dimension as a point on a line representing the cellular axis. The cell diameter is fixed at $40 \mu \mathrm{m}$. Fig. 1 shows the schematic of the model for studying the single centrosomal dynamics. As discussed before, the forces include astral MT buckling induced push (inwards, away from the cell cortex), cortical dynein motor mediated pull (outwards, toward the cell cortex), and a steric force to confine the CS within the cell cytoplasm. The model's primary focus is to evaluate the interplay between these forces that dictate the force landscape of CS and bring about its positioning at the cell center.

Fig. 3(a) shows centrosomal dynamics with initial position at $t=0 \mathrm{~s}$ of the CS at $5 \mu \mathrm{m}, 15 \mu \mathrm{m}, 20 \mu \mathrm{m}, 25 \mu \mathrm{m}$ and $35 \mu \mathrm{m}$ along the cellular axis in a cell of diameter $40 \mu \mathrm{m}$. We observe in Fig. 3(a) that the CS stably positions itself at the cell center, irrespective of its initial position at $t=0 \mathrm{~s}$. For initial positions at $5 \mu \mathrm{m}$ and $35 \mu \mathrm{m}$, the CS is seen to move toward the cell center from the very beginning. As the CS is very close to the cell cortex at $t=0 \mathrm{~s}$, short astral MTs emanating from it can easily reach the cortex. This interaction generates strong MT buckling mediated pushing force (since it is inversely proportional to the square of MT length acc. to (5)), causing the CS to move toward the cell center. The astral MTs from the CS on the other side (toward the distal cell cortex) have to grow to relatively longer lengths to reach the cell cortex. Thus, their contribution to the force landscape remains negligible at this point. As its motion progresses toward the cellular interior, the distance between the CS and the distal cell cortex reduces. As the distal cell cortex becomes more accessible to the astral MTs, their contribution to the force landscape of the CS gains significance. Since the net MT length remains comparatively long when the astral MTs reach the distal cell cortex, the buckling mediated force becomes less intense (refer to (5)), and their contribution to the force landscape thins out. As the CS moves toward the cell center and the distance between the CS and the distal cortex shrinks further, the buckling-induced force from the distal cell cortex becomes more prominent. The increasing buckling force from the distal cortex competes with the force contribution from the proximal cell cortex while maintaining a directed motion toward the cell center. At the cell center, the forces from the cell cortex on both sides of the CS become comparable. This eventually leads to attaining the force balance, thus stabilizing the CS at the cell center. The same also holds for the CS initially placed right at the cell center at $t=0 \mathrm{~s}$. As seen in 3(a), the CS starting at $20 \mu \mathrm{m}$ at $t=0 \mathrm{~s}$ is acted upon by the competing force landscapes from both sides that mediate its stay at the cell center. For the initial positions of the CS at $15 \mu \mathrm{m}$ and $25 \mu \mathrm{m}$ at $t=0 \mathrm{~s}$, it moves toward the cell cortex for a period of $\sim 300 s$ at the beginning followed by movement toward the cell center. The initial placement of the CS at intermediate points along the cellular axis $(15 \mu \mathrm{m}$ and $25 \mu \mathrm{m})$ causes the astral MTs emanating from the CS to grow to greater lengths to reach the proximal cell cortex. This causes the buckling-induced force from the proximal wall to become less intense. The dynein-mediated contribution to the force landscape stays uncompromised as the astral MTs can ultimately reach the cell cortex and bind to the dynein motors. Therefore, the force landscape is dominated primarily by the dynein motor mediated pulling force that pulls the CS toward the proximal cell cortex during this initial period of $\sim 300 s$ as shown in Fig. 3(a). But this movement of the CS toward the cell cortex reduces the distance between the CS and the proximal wall, thus intensifying the buckling induced force. These strong inward forces acting on the CS then mediate its movement toward the cell center.

To establish the roles of individual force contributors in positioning the CS at the cell center, we systematically exclude force contributors from our model one by one and examine their impact. We begin by setting the buckling amplitude $A_{b u c k}$ to zero on both sides of the CS. The CS does not experience any pushing force from the cell cortex on either side in such a scenario. The cortical dynein motors mediate the only force that act on it. Fig. 3(b) shows the resultant centrosomal dynamics when buckling mediated forces are absent on both sides of the CS. For the CS initially placed anywhere along the cellular axis other than the cell center, the astral MTs reach the proximal cell cortex faster and more significantly in numbers than the distal cell cortex. This exerts strong motor-mediated pulling force on the CS, directed toward the proximal cell cortex, away from the cell center. Thus, in the absence of buckling induced counter pushing force, the CS is dragged to the proximal cell periphery where the steric forces stabilize the CS. For the CS placed at the cell center at $t=0 \mathrm{~s}$, the astral MTs are equally likely to reach the cell cortex on either side of the CS. Thus, the CS has an equal probability of getting dragged to either the left or the right cell periphery when initially placed at the cell center. This is indicated in Fig. 3(b) by the large errorbars in the resulting dynamics of the CS starting at $20 \mu \mathrm{m}$. Therefore, even though it may seem that dynein mediated pulling forces from both sides of the CS should counter each other and achieve a force balance, we find from Fig. 3(b) that dynein mediated forces are not the main contributors in the stable positioning of the CS at the cell center.

We now proceed by excluding the force contribution of the cortical dynein motors on both sides of the CS such that no pulling force act on it from either side. Fig. 3(c) shows centrosomal dynamics obtained by setting cortical dynein motor density to zero on both sides. The CS is seen to position itself at the cell center stably. Since, 
bioRxiv preprint doi: https://doi.org/10.1101/2021.10.04.463000; this version posted October 4, 2021. The copyright holder for this preprint (which was not certified by peer review) is the author/funder, who has granted bioRxiv a license to display the preprint in perpetuity. It is made available under aCC-BY-NC-ND 4.0 International license.

(a)

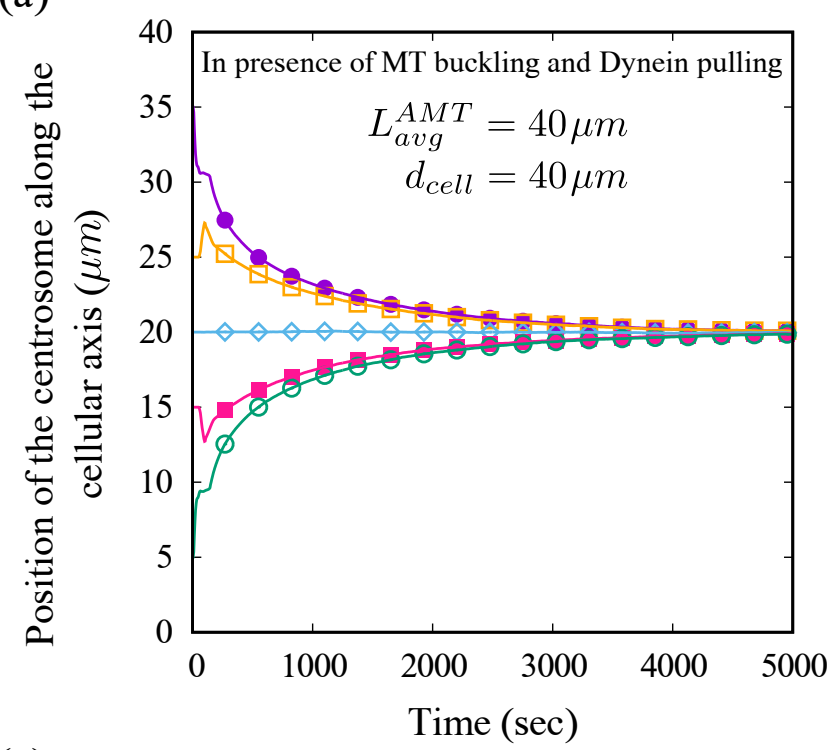

(c)

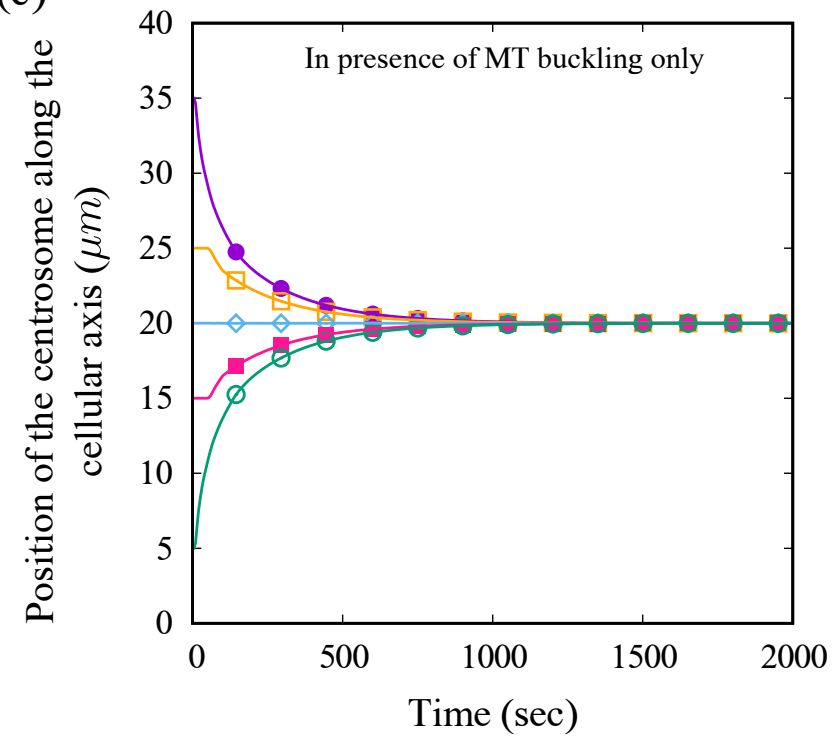

(b)

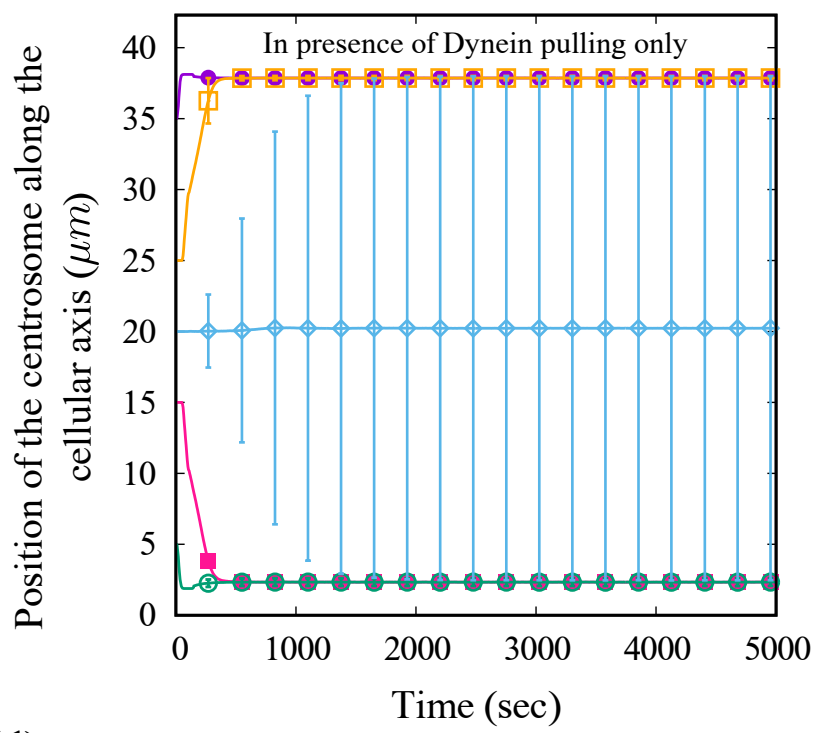

(d)

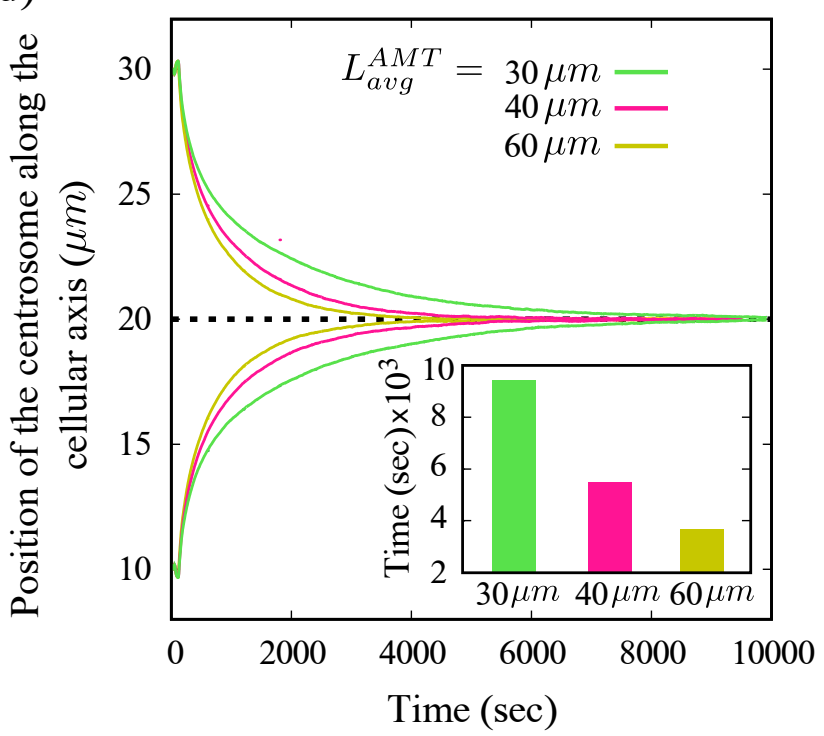

FIG. 3: Variation in the position of CS over time is plotted for different initial positions at $t=0 \mathrm{~s}$ showing the role of astral MT buckling, cortical dynein motors, and average astral MT length. While figures (a), (b), (c) show CS dynamics for initial position at $5 \mu \mathrm{m}, 15 \mu \mathrm{m}, 20 \mu \mathrm{m}, 25 \mu \mathrm{m}$ and $35 \mu \mathrm{m}$ at $t=0 \mathrm{~s}$, figure (d) shows the same for CS initially placed at 10 $\mu \mathrm{m}$ and $30 \mu \mathrm{m}$. (a) The mean CS position along the cellular axis (in $\mu \mathrm{m}$ ) is plotted versus time (in sec) for cell diameter $40 \mu \mathrm{m}$ with astral MT avg. length $40 \mu \mathrm{m}$. (b) CS dynamics simulated without MT buckling (Abuck=0). Standard deviation in the data obtained with 1000 ensembles indicates that CS's position at the cell center is not stable without MT buckling. (c) CS dynamics simulated without cortical dynein $\left(k_{d y n}=0\right)$ showing that CS positioning at the cell center is dominated by force due to buckling of astral MTs. (d) The CS position plotted versus time for varying average MT length; inset: a bar graph showing duration required for CS positioning for various average MT lengths; faster positioning of the CS at the cell center is achieved by longer MTs.

only the buckling-induced force pushes the CS inwards toward the cell center, there is no counter force to oppose this movement due to the absence of cortical dynein motors on both sides of the CS. Thus, a CS starting at any point along the cellular axis except the cell center would be pushed toward the cell center under the net buckling force. Primarily, the proximal cell cortex medi- ates stronger buckling force compared to the distal cell cortex. But as the CS approaches the cell center, the buckling-induced force from the distal cortex intensify and become more prominent. With the CS at the cell center, the buckling mediated pushing force from either side of the CS can compete with each other. Fig. 3(c) shows that this eventually leads to a force balance at the 
cell center that stabilizes the CS there. Thus, it is evident that astral MT buckling can position the CS at the cell center without the dynein motors' assistance, as shown in Fig. 3(c). Therefore, we conclude that the astral MT buckling induced force is responsible for positioning the $\mathrm{CS}$ at the cell center during Interphase.

\section{CS rapidly positions at the cell center with longer astral MTs}

In this section, we change the astral MT average length $L_{a v g}^{A M T}$ keeping the cell diameter constant to examine its impact on CS positioning. We start by altering the dynamic instability parameters of the astral MTs as they determine the $L_{a v g}^{A M T}$ by eq. (1). We vary their velocities of growth and shrinkage while keeping their frequencies of catastrophe and rescue fixed (shown in Table I). The $L_{a v g}^{A M T}$ was varied with respect to the cell diameter $d_{c e l l}$ by keeping it at $30 \mu m\left(<d_{\text {cell }}\right), 40 \mu m\left(=d_{\text {cell }}\right)$ and $60 \mu \mathrm{m}\left(>d_{\text {cell }}\right)$. Fig. 3(d) showcases the centrosomal dynamics obtained when the CS is initially placed at $10 \mu \mathrm{m}$ and $30 \mu \mathrm{m}$ along the cellular axis for varying $L_{a v g}^{A M T}$. The results indicate that the time required for the CS to a position at the cell center decreases when astral MTs present in the system have higher $L_{a v g}^{A M T}$. Since the centrosomal dynamics is governed by forces originating from the astral MTs' interaction with the cell cortex, a higher $L_{a v g}^{A M T}$ ensures stronger interaction between them, thus mediating faster positioning of the CS. The bar graph in the inset of Fig. 3(d) indicates the average time duration required by the CS to position at the cell center for differing astral MT average lengths. It explicitly shows that the positioning of the CS at the cell center is faster for astral MTs with a higher average MT length.

\section{CS position depends on the initial condition in presence of short astral MTs}

This section discusses the centrosomal positioning when astral MTs are relatively shorter with respect to the cell diameter. Fig. 4 showcases the results obtained from the model for a cell of diameter $60 \mu \mathrm{m}$ with $L_{a v g}^{A M T}$ set at $40 \mu \mathrm{m}$. Fig. 4(a) shows that the centrosomal positioning does not occur at the cell center for these parameters. It is important to note here that astral MT buckling amplitude and linear density of cortical dynein motors, denoted by $A_{b u c k}$ and $k_{d y n}$, respectively, are kept unchanged as in the previous sections (see Table I). Fig. 4(a) shows the resultant CS dynamics for its initial placement at $5 \mu \mathrm{m}$, $25 \mu \mathrm{m}, 30 \mu \mathrm{m}, 35 \mu \mathrm{m}$ and $55 \mu \mathrm{m}$ along the $60 \mu \mathrm{m}$ long cellular axis. The CS is placed very close to their respective proximal cell cortex for initial positions $5 \mu \mathrm{m}$ and $55 \mu \mathrm{m}$. Therefore, many short astral MTs can easily reach the cell cortex generating strong buckling-induced force. This causes the CS to initially move toward the cellular interior as shown in Fig. 4(a).
The movement of the CS toward the cell center increases the distance between the CS and the proximal cell cortex. The increased distance reduces the intensity of the buckling-mediated pushing force allowing the dynein-mediated pulling force from the proximal cell cortex to take precedence. These pulling forces pull the CS back toward the cell cortex, preventing its journey toward the cell center. The initial distance between the CS and the proximal cell cortex is greater for the CS starting at intermediate points along the cellular axis (at $25 \mu \mathrm{m}$ and $35 \mu \mathrm{m}$ ). This allows the dynein-mediated pulling force to play the dominant role during the initial movement of the CS. Thus, the CS is initially pulled toward the proximal cell cortex, as shown in Fig. 4(a). Again as the CS approaches the cortex, the buckling force intensifies, hindering their motion toward the cortex. Since the $L_{a v g}^{A M T}$ is short compared to the cell size, the force contribution from the distal cell cortex remains primarily insignificant. Therefore, the force landscape of the CS comprises an interplay between the pulling and the pushing forces mediated mainly by the proximal cell cortex. Eventually, a force balance results from this interplay stabilizing the CS away from the cell center.

Exciting things happen when CS starts precisely at the cell center. In this case, there is an equal probability of the CS to position on either side of the cell center, shown by the large errorbars in its dynamics in Fig. 4(a). The astral MTs emanating from either side of the CS have to grow equal distances to reach the cell cortex on both sides. Since the cell size is large in comparison to the $L_{a v g}^{A M T}$ of the astral MTs, the length to which they must grow to reach the cell cortex on either side is long. This reduces the buckling-induced force, and cortical dynein motors dominate the net force on the CS at the cell center. In the previous section from Fig. 3(b), we have concluded that the pulling force cannot balance the net force on the CS at the cell center. Therefore, the CS is dragged away from the cell center when the astral MTs contact any cell cortex. This motion is directed toward that cell cortex which allows the MTs to establish connection faster. It is important to note that the astral MTs are equally likely to contact the right or left cortex. Fig. 4(a) shows that the CS can position on either side of the cell center with equal probability when it starts at the cell center.

In Fig. 4(b), we tune the model such that the interaction between microtubule and cortex is weak on one side than the other. Such a possibility may arise for an elongated cell or when the MT array is asymmetric. In the model, forces are switched off on the left half of the cell (setting the buckling amplitude $A_{\text {buck }}$ and dynein density $k_{d y n}$ to zero) while keeping the right half undisturbed. The CS in Fig. 4(b), initially placed within the left half (at 5 and $25 \mu \mathrm{m}$ ), is seen to migrate toward the right half indicating that it is under the influence of an effective attraction originating from the right cortex. Moreover, irrespective of the initial position, CS stabilizes on the right half of the cell. Thus, the force bal- 
(a)

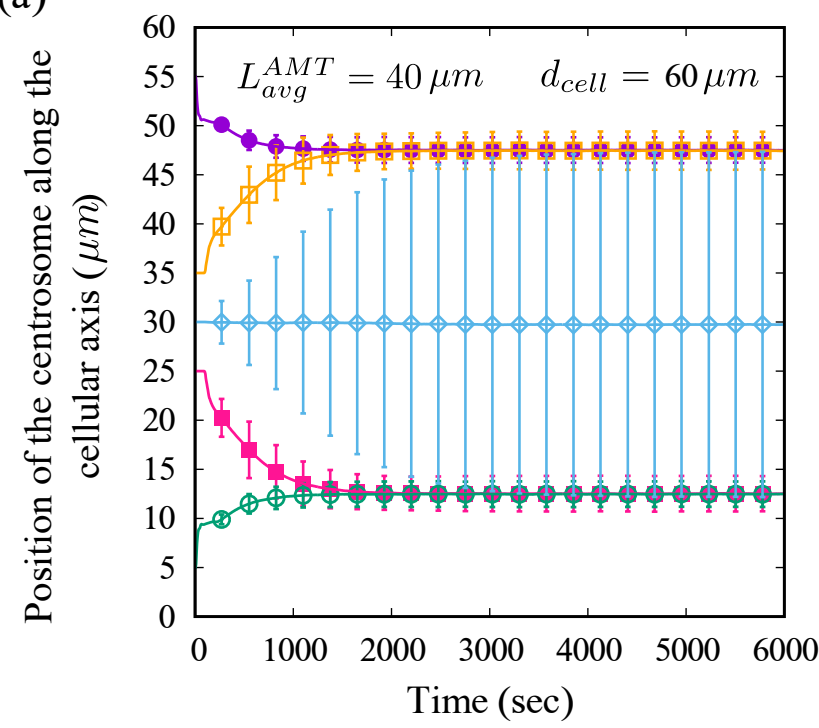

(b)

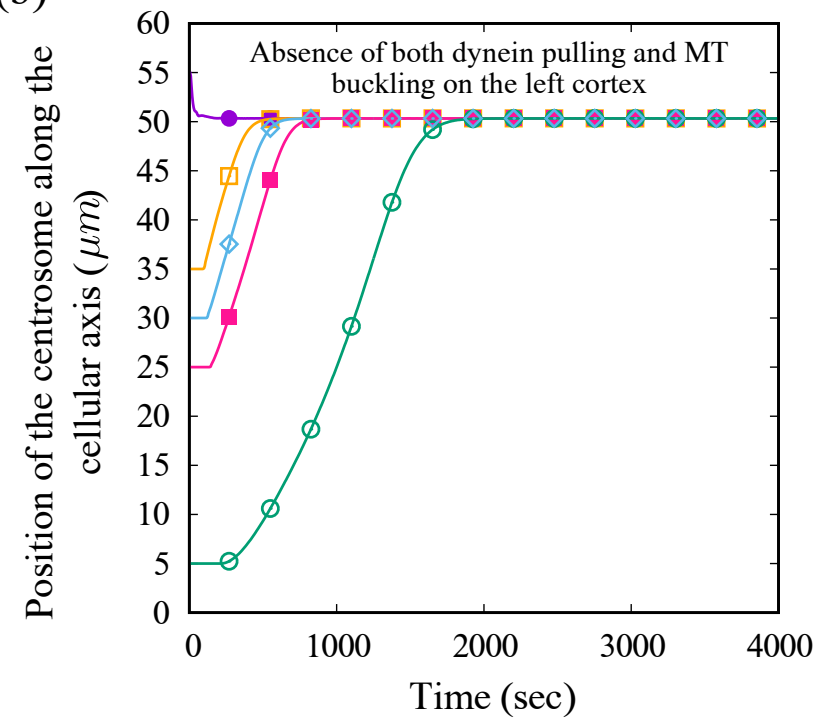

FIG. 4: Dynamics of a single CS for short MTs with initial positions at $5 \mu m, 25 \mu m, 30 \mu m, 35 \mu m$ and $55 \mu m$. (a) The mean position of the CS with standard deviation plotted against time for cell diameter $=60 \mu \mathrm{m}$ and avg. MT length $=40 \mu \mathrm{m}$. (b) Centrosomal position in absence of MT buckling and dynein pull from the left cortex (lower half of the plot) while the right cortex (upper half of the plot) is kept unmodified.

ance is independently established by pushing and pulling forces associated with the cell cortex on the right.

The dynamics of CS discussed in this context (Fig. 4(a)) can be modified by altering the force parameters. E.g., the buckling amplitude $A_{b u c k}$, cortical dynein density $k_{d y n}$, have a crucial effect on the CS position [discussed in Appendix I].

\section{B. Dynamics of Bipolar Spindle}

\section{Astral and interpolar MTs together with cortical dynein robustly determine spindle kinetics during Anaphase B}

During mitotic metaphase, the bipolar spindle poles maintain an approximately stable separation between them. The interpolar distance increases as the cell enters anaphase. A gradual slowdown follows this increase before a stable interpolar separation is achieved. The duration of the spindle elongation and subsequent positioning is observed to be in the range of 150-200 seconds in C. elegans [41] where the astral MTs play the pivotal role.

Fig. 5 shows the resultant spindle dynamics obtained from our model. Initially, the poles are placed symmetrically with respect to the cell center separated by a distance of $10 \mu \mathrm{m}$ (initial positions of individual poles are $20 \pm 5 \mu \mathrm{m}$ on either side of the cell center) in a $40 \mu \mathrm{m}$ cell. In Fig. 5(a), the variation in the interpolar distance is plotted versus time for two separate scenarios: with the (i) participation of IPMTs only, and (ii) participation of both IPMTs and the astral MTs. The elongation of the spindle mediated by the IPMTs only, as shown in Fig. 5(a), continues to grow linearly and does not saturate. However, a closer look into the data indicates a faster separation in the early stages (see inset of Fig. 5(a) for interpolar separation in the first $20 \mathrm{sec}$ ). To address this, we investigate the IPMT overlap dynamics and the sliding velocity of the kinesin-5 motors that drive elongation of the spindle, as shown in Fig. 5(b). The crosslinking kinesin-5 motors maintain the overlap between the antiparallel IPMTs. In our model, the number of kinesin-5 motors actively participating in the elongation process is proportional to the IPMT overlap in the spindle interzone. The velocity of these motors along the IPMTs equals the rate at which IPMTs are being slid apart, given by $V_{\text {sliding }}$. From the Fig. $5(\mathrm{~b})$, we observe that the motor velocity $V_{\text {sliding }}$ falls as the overlap $L(t)$ falls, reaching a small value much less than their maximum unloaded velocity at $0.07 \mu \mathrm{m} / \mathrm{s}$. This points to the kinesin-5 sliding motors operating near their stall condition as the IPMT overlap decreases. The phenomenon is reported earlier (12) where the motor velocity $V_{\text {sliding }}$ is seen to depend on the dynamic overlap $L(t)$ between the antiparallel IPMTs. The fall in the IPMT overlap thus accompanies decreasing sliding rates of kinesin-5 motors. As the IPMT sliding rate suffers a setback due to reducing overlap, the elongation of the spindle cannot proceed at the desired pace after the initial separation $(\sim 5$ sec). Also, since the IPMT overlap does not diminish entirely, as shown in Fig. 5(b), the sliding motors never actually stall completely, continuing the elongation process. Therefore, the mechanism mediated by IPMTs only helps push the poles apart but fails to give them a stable separation within the cell cytoplasm. In contrast, when 
(a)

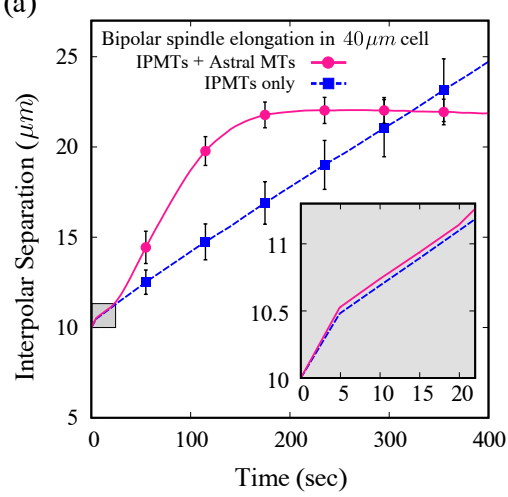

(b)

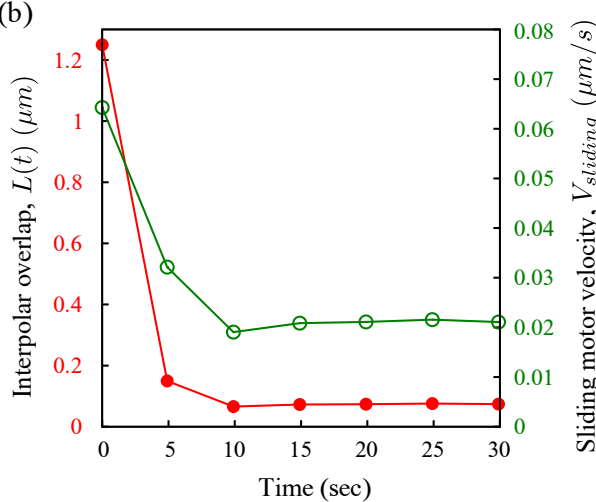

(c)

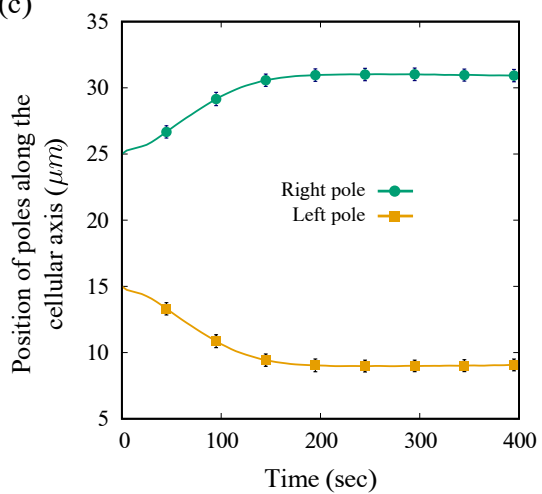

FIG. 5: Bipolar spindle elongation during anaphase B. (a) The mean separation between the two poles constituting the bipolar spindle is shown for two cases when only the IPMTs participate and when the astral MTs and the IPMTs participate together. The cell diameter is kept at $40 \mu \mathrm{m}$ with initial interpolar separation at $10 \mu \mathrm{m}$. The error bars signify the standard deviation. Inset: Bipolar spindle kinetics is shown for the first $\sim 20 \mathrm{~s}$. The elongation up to this time frame is primarily mediated by IPMTs and is seen to decrease after the initial $\sim 5 \mathrm{~s}$ as an impact of decreasing antiparallel IPMT overlap. (b) The antiparallel IPMT overlap, $L(t)$, is plotted versus time with the corresponding sliding velocity, $V_{\text {sliding }}$, of kinesin-5, which drive the IPMT mediated bipolar elongation. (b) Mean position of individual poles is shown as a function of time when the IPMTs and the astral MTs participate together.

the astral MTs participate alongside the IPMTs, the interpolar separation is seen to increase and then saturate as observed in the previous experimental studies [41].

Upon comparing the elongation dynamics in Fig. 5(a) obtained for scenarios (i) and (ii) mentioned before, we find an initial overlap between them highlighted by the gray area. This spans the first $\sim 20 s$ when the spindle elongation is governed primarily by the IPMTs. During this period, most astral MTs cannot reach the cell cortex and contribute negligibly to the net force. Beyond this time frame, when the astral MTs actively participate, the poles are seen to elongate relatively faster than when IPMTs participate alone.

The astral MTs grow to reach the cell cortex and interact via MT buckling and cortical dynein motors binding to the astral MTs. The interpolar separation is small at the beginning of spindle elongation, while the distance between each pole and the proximal cortex is significant. The astral MTs emanating from each pole must grow long enough to reach the proximal cortex. As MTs reach the respective cortex, the corresponding buckling force (inversely proportional to the square of MT length (5)) starts to build up and push weakly on the respective individual centrosomes. At the same time, dynein-mediated pulling force (proportional to the cortical dynein density (4)) act on each centrosome and dominate over the buckling force. Therefore, the net force on the spindle imparted by the cortical dynein motors controls the initial elongation of the spindle resulting in a steady increase in the interpolar separation. As the separation between the poles increases, the distance between each pole and proximal cortex also decreases. Thus, a large number of astral MTs can reach the cortex, and many of them are short in length. This leads to a change in the net force on the centrosome as the buckling force mediated by shorter as- tral MTs compensate the dynein-mediated pulling force. This hinders the elongation process of the spindle. As shown in Fig. 5(a), the elongation rates gradually decrease to zero, and the interpolar separation saturates. At this stage, a force-balance between dynein pull and MT buckling is achieved, preventing further elongation of the spindle. This is in contrast to the monotonic extension of the spindle without any saturation when only IPMT-mediated force contributes.

Fig. 5(c) shows the explicit movement of individual poles when both IPMTs and astral MTs participate. This movement corresponds to the net interpolar separation shown in Fig. 5(a). Note that, in this case, the poles were initially placed symmetrically about the cell center. The movement and final positioning of individual poles are also symmetric with respect to the cell center. The symmetry in spindle positioning is obtained even when the initial position of the spindle is not at the cell center (additional details are given in Appendix II).

\section{Astral MTs and cell size crucially regulate the rate of elongation and spindle length}

Bipolar spindle elongation depends on astral MTs' interactions with the cell cortex, which depends on the dynamic instability at their plus ends. The dynamic instability is determined by characteristic parameters $v_{s}, v_{g}, f_{\text {cat }}$ and $f_{\text {res }}$, which regulate the average length of MTs (see 1). In this section, we vary the average length of the astral MTs $\left(L_{a v g}^{A M T}\right)$ to explore its impact on the elongation and positioning of the bipolar spindle. The average length of the IPMTs is kept unchanged. Fig. 6(a) shows the spindle elongation dynamics obtained for $L_{a v g}^{A M T}=5 \mu m, 10 \mu m, 40 \mu m$ and $60 \mu m$ in a cell of 
bioRxiv preprint doi: https://doi.org/10.1101/2021.10.04.463000; this version posted October 4, 2021. The copyright holder for this preprint

(a)

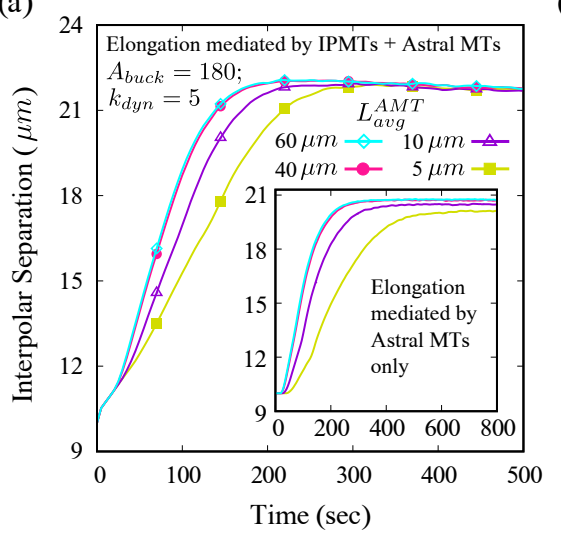

(b)

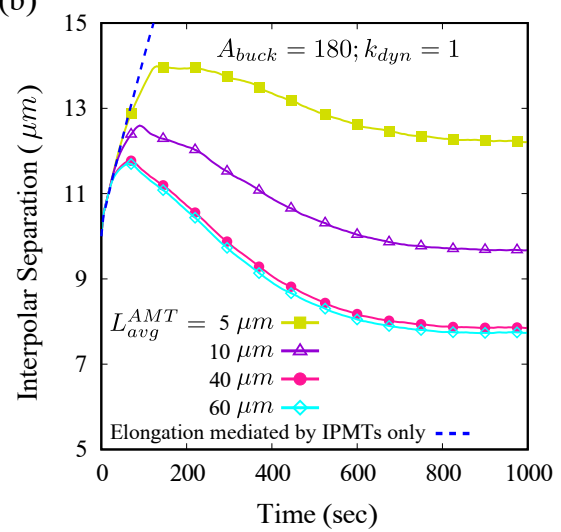

(c)

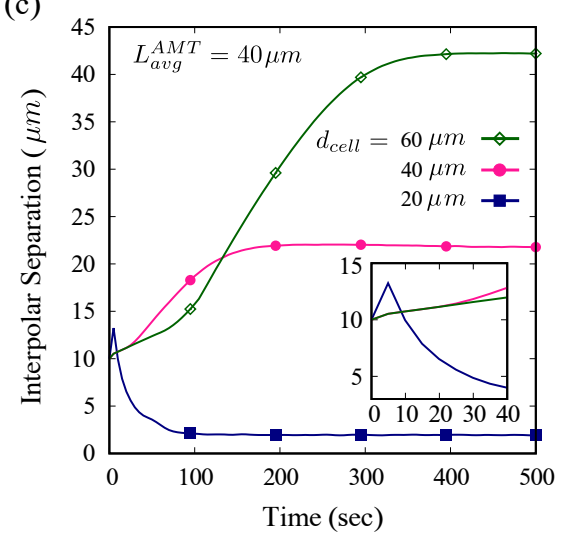

FIG. 6: The impact of varying MT average length and cell diameters on bipolar spindle elongation. (a) The mean interpolar separation versus time shown for a $40 \mu \mathrm{m}$ cell with average astral MT length of $5 \mu \mathrm{m}, 10 \mu \mathrm{m}, 40 \mu \mathrm{m}$ and $60 \mu \mathrm{m}$; Inset: The mean separation obtained without IPMTs and only via astral MTs for the same set of MT length. (b) Interpolar separation similar to (a) but for small dynein density. (c) Kinetics of interpolar separation for varying cell diameters $20 \mu m, 40 \mu m$ and $60 \mu \mathrm{m}$, and average astral MT length $40 \mu \mathrm{m}$; Inset: Spindle dynamics shown during the first $40 \mathrm{~s}$ for differing cell diameters (as in (c)).

diameter $40 \mu m$. When $L_{a v g}^{A M T}$ is less than the cell diameter (at $5 \mu \mathrm{m}$ and $10 \mu \mathrm{m}$ ), the spindle elongation rates appear to depend on the $L_{a v g}^{A M T}$ with shorter MTs taking longer to achieve a stable interpolar separation. For $L_{a v g}^{A M T}(40 \mu m$ and $60 \mu m)$ greater or equal to cell diameter; however, the elongation rates are fairly comparable. Steady-state position and extension of the spindle appear to be the same, irrespective of the variation in the average MT length.

Notice that the spindle length is small in the early stage of the pole separation, and the distance between the poles and their respective proximal cortex is large. Thus, the astral MTs emanating from a pole must grow long to reach the cell cortex. Long astral MTs cannot generate strong buckling force (see (5)), causing the net force on each centrosome to be dominated by dynein pull from the cortex. This causes a steady elongation of the spindle beyond the initial $\sim 20 s$ when elongation is primarily mediated by the IPMT-sliding (see Fig. 6(a)). Now, for a given separation between a pole and the proximal cortex, longer astral MTs reach the cell cortex faster than shorter MTs. This causes the spindle to elongate at a faster rate when $L_{a v g}^{A M T}$ is greater. Hence, the spindle's elongation rate varies with average MT length, as shown in Fig. 6(a). For astral MTs with an average MT length greater or equal to the cell diameter, the elongation rates almost coincide as the force contribution from the long astral MTs are very similar. As the spindle elongates, the distance between the poles and their respective proximal cortex shrinks. This allows shorter astral MTs to reach the cortex, increasing the buckling-induced force on the spindle, thus hindering the steady elongation of the spindle. With increasing buckling force, the net force on the spindle is no longer dominated by the cortical dynein motors. Spindle elongates gradually and then saturates when the pushing and pulling forces balance each other.
In the inset of Fig. 6(a), the spindle elongation dynamics for different $L_{a v g}^{A M T}$ is plotted without IPMT force. With only astral MTs present in our model, we observe that the extent to which the spindle elongates depends on the average length of the astral MTs. The final interpolar separation almost coincides for $L_{a v g}^{A M T}$ greater or equal to the cell diameter. For small values of $L_{a v g}^{A M T}$, the final interpolar separation is relatively smaller and depends on the magnitude of $L_{a v g}^{A M T}$. The result in the inset of Fig. 6(a) when compared to the main plot in Fig. 6(a), reveals that IPMTs not only determine the final spindle length but also keep it relatively independent of the average length of the astral MTs.

In Fig. 6(b), the spindle elongation obtained for various $L_{a v g}^{A M T}$ is plotted for a small dynein motor density on the cell cortex. The dotted line shown in Fig. 6(b) is the reference of spindle elongation when only IPMTs are present. The solid lines with points are for varying $L_{a v g}^{A M T}$ and consider both IPMTs and the astral MTs. The interpolar separation shows that the spindles initially elongate consistently, followed by contraction. The astral MTs, in contact with the cortex, generates both buckling and dynein-induced force. The buckling force becomes more influential here since the dynein density is low. Consequently, the buckling force dominates and push the poles away from the cortex, reducing interpolar separation. From this position, the astral MTs with higher $L_{a v g}^{A M T}$ can reach the cortex faster and thus, buckle earlier. Therefore, the resultant spindle length with higher $L_{a v g}^{A M T}$ is smaller when dynein is impaired in the cortex.

We further explore the spindle elongation dynamics by varying the cell diameter while keeping the $L_{a v g}^{A M T}$ constant at $40 \mu \mathrm{m}$. The cell diameter is varied in steps of $20 \mu \mathrm{m}$ starting from $20 \mu \mathrm{m}$ till $60 \mu \mathrm{m}$. The spindle evolution in Fig. 6(c) for cell diameters $40 \mu \mathrm{m}$ and $60 \mu m$ shows similar elongation patterns that have been 
discussed so far. The primary IPMT mediated elongation continues for a longer duration in the $60 \mu \mathrm{m}$ cell than the $40 \mu \mathrm{m}$ cell. Also, the spindle elongation occurs to a much larger extent in the former, before the saturation sets in. This is due to the astral MTs that take longer to reach the cell cortex in a larger cell (with diameter $60 \mu \mathrm{m}$ ), slowing down the initial pole separation. The same factor also delays the exertion of buckling mediated pushing force on the poles, causing the spindle to elongate further. As for the cell with diameter, $20 \mu \mathrm{m}$, the astral MTs (with $L_{a v g}^{A M T}=40 \mu \mathrm{m}$ ) reach the cortex early and strongly pull on the poles outward, via cortical dynein motors. The cortical pulling causes the spindle to rapidly elongate in the beginning (see Fig. 6(c)) for the first $\sim 10 s$ (see inset of Fig. 6(c)). As the distance between the cortex and the poles shrinks, buckling force pushes the poles inward, hindering the steady elongation process. The cell size being small compared to $L_{a v g}^{A M T}$, the buckling force becomes very strong after only a small elongation of the spindle, causing the poles to move toward each other steadily. In fact, for the smallest cell considered here (diameter $20 \mu \mathrm{m}$ ), the buckling force grows so intense that the spindle effectively collapses (see Fig. 6(c)).

\section{Bipolar spindle grows abnormally long in absence of buckling force due to astral MTs}

In this section, we exclude the MT buckling force and study the spindle separation kinetics. In the absence of buckling, IPMT sliding force push the poles apart while cortical dynein motors pull the poles toward the cortex via astral MTs. The resulting spindle elongation dynamics with and without buckling force, shown in Fig. 7(a), indicate that the spindle separation is abnormally long in the absence of MT buckling force. As the poles approach near the cell cortex on their respective sides (see 7(b)), they encounter steric force from the cortex that restricts further spindle elongation. Thus, spindle length saturates close to the cell size (diameter). This is in sharp contrast to the typical spindle, as shown in Fig. 7(b). The spindle under normal conditions is relatively smaller, and poles are suitably positioned inside the cell. The buckling force thus plays a decisive role in placing the spindle poles within the cell cytoplasm and maintaining the spindle length.

\section{Bipolar spindle fails to elongate and position in the absence of cortical dynein}

This section excludes the dynein-mediated pulling force to examine their impact on the spindle elongation dynamics. Under such a condition, the IPMTs slide and elongate the spindle under such a condition while the astral MT buckling force shortens the spindle. Fig. 7(c) shows the spindle dynamics in the presence and absence of dynein-mediated pulling force. The primary IPMTmediated elongation can be seen in the beginning as the spindle elongates during the first $\sim 20 \mathrm{~s}$. Once the astral MT buckling force act on the poles, the elongation of the spindle is hindered. The inset in Fig. 7(c) shows the dynamics of individual poles with time. The poles move toward each other and tend to collapse, but the steric force between them avoid this possibility. Fig. $7(\mathrm{~d})$ shows dynamics of individual poles when the dynein-mediated pulling force acts from one cortex only. Therefore, besides the IPMTs and the buckling forces as before, there is a contribution from the dynein-mediated pulling force on the spindle only from the left cortex (i.e., $y \sim 0$ in Fig. $7(\mathrm{~d}))$. The opposite cortex on the right (i.e., $y \sim 40$ in Fig. $7(\mathrm{~d})$ ) has no cortical dynein motors and, therefore, offers no pulling force. In Fig. $7(\mathrm{~d})$, we observe that the left pole can elongate and position normally, as found earlier. On the other hand, the right pole is pushed toward the cell interior due to buckling force from the right cortex. Finally, due to IPMTs sliding force, a force balance is achieved between the opposite poles that position the spindle asymmetrically about the center of the cell.

\section{DISCUSSION}

Positioning of the CS, like many other cellular organelles, is crucial for cellular functioning. While the CS position during Interphase is primarily dominated by MT buckling mediated pushing force, the bipolar spindle dynamics during Anaphase B is a consequence of the interplay between the buckling mediated pushing force and the dynein induced pulling supported by the IPMT mediated sliding force. Our model for positioning of the single CS reveals that it is strongly driven by the cortical pushing force caused by the buckling of astral MTs interacting with the cortex. However, in the presence of short astral MTs or within an elongated cell, the mechanism allows for positioning the CS away from the cell center. The stable positioning of the CS can be controlled by regulating the net buckling of the astral microtubules and the cortical dynein density [see Appendix I]. A mechanism similar to centrosome positioning is at work in fission yeast $(S$. pombe) cells during Interphase. The nucleus in $S$. pombe cells which is the microtubuleorganizing center during Interphase, is positioned at the cell center by the microtubules emanating from its north and south poles. Experimental studies have shown that this mechanism is pushing force dominated [23]. Since our model reveals dominance of pushing force for centrosomal positioning in one dimension, the same model can also provide a qualitative understanding of the nuclear positioning at the cell center in cylindrical fission yeast cells during Interphase [23]. Even though the stable positioning of the single CS is influenced by MT buckling, the dynein density at the cortex can play a significant role in altering the $\mathrm{CS}$ position. Without dynein, the CS invariably positions at the cell center, and therefore 
(a)

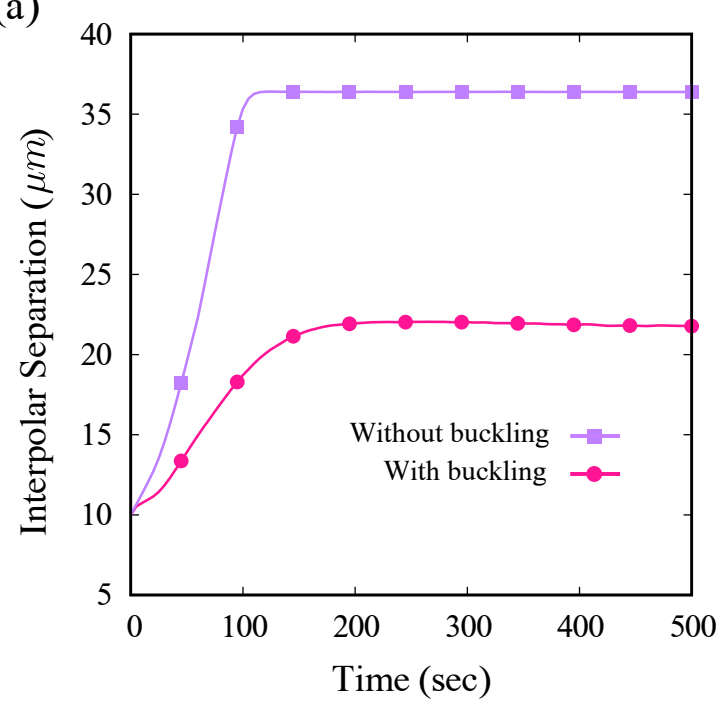

(c)

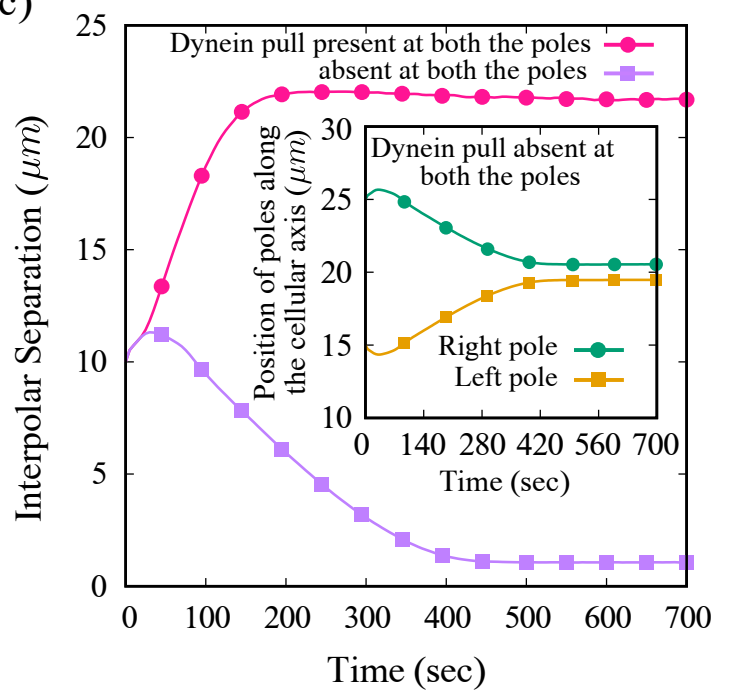

(b)

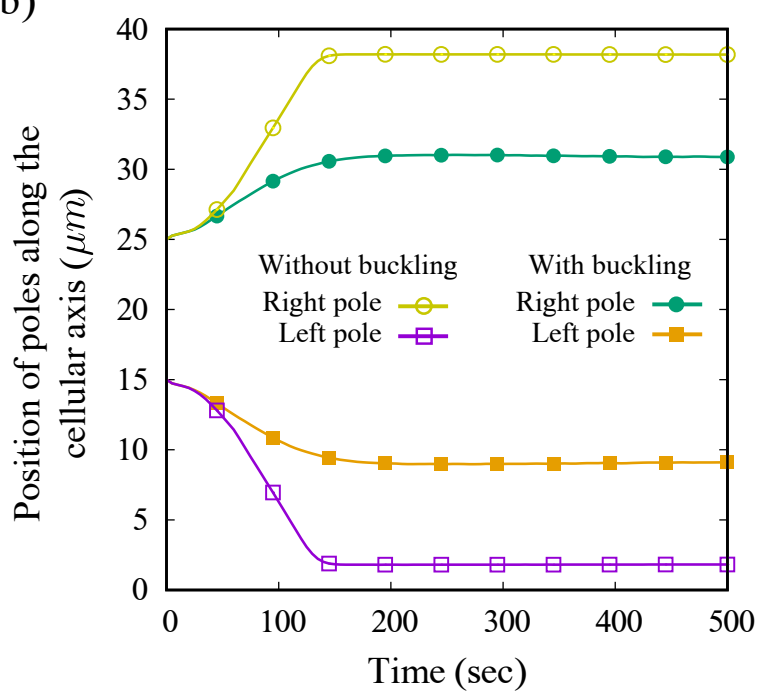

(d)

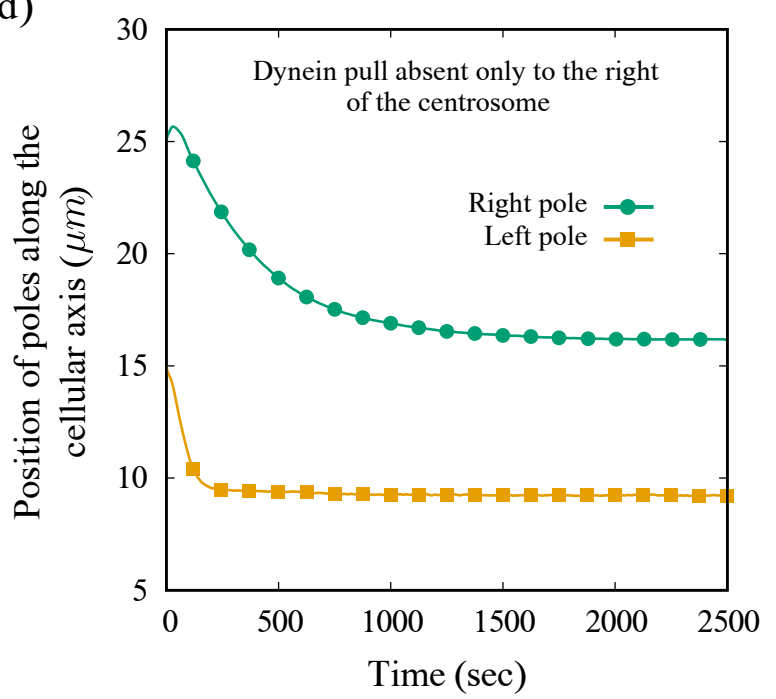

FIG. 7: Bipolar spindle elongation and the role of dynein mediated pulling and buckling mediated pushing forces. (a) The mean interpolar separation is plotted versus time in the presence and absence of astral MTs mediated buckling. (b) The mean positions of the individual poles corresponding to (a). (c) The interpolar separation is plotted versus time with and without dynein-mediated cortical pull via astral MTs. Inset: the mean positions of individual poles in the absence of dynein-mediated force on the poles. (d) The mean positions of the individual poles in the absence of dynein-mediated pull on the right pole.

altered positioning of the CS cannot be explained with MT buckling alone. Thus a robust positioning of the CS requires MT buckling as well as dynein pull from the cortex.

Proper elongation and subsequent positioning of the bipolar spindle are crucial milestones for the cell to achieve faithful chromosomal segregation and cytokinesis during mitosis. The cortical dyneins are essential for spindle elongation. They help stretch the spindle by applying pulling force from the cortex, which is critical in anaphase B. However, a stable position of the metaphase spindle may be perturbed by a strong dynein pull from the cortex. Previous studies emphasized that cortical dynein pulling is anti-centering in nature, meaning that they tend to move the spindle away from the cell cen- ter $[9,34]$. Yet, cortical dynein facilitates the force balance in unison with the antagonizing buckling mediated pushing force. The uniform MT-cortex interactions regulate a robust and symmetric spindle positioning in the cell mid-zone along the cellular axis.

The minimal model considered here has limitations. For instance, uniform density of dynein on the cortex considered in the model cannot account for asymmetric cell division [19, 20] which could arise from the asymmetric positioning of the bipolar spindle [see Appendix II]. Also, we employed a mean-field approach while evaluating the force exerted by each dynein motor and astral MTs interacting with the cortex. Additional features may arise if stochastic binding-unbinding of the motors is considered. In addition, catch-bond-like binding of dynein mo- 
tors [21] to the astral MTs with force-dependent attachment and detachment rates of the unbound and bound motors, respectively, may be another interesting aspect to consider in the future studies [57]. Another aspect ignored in this model is the absence of force, a generating mechanism involving the cytoplasm [9]. Experimental evidence accounts for cytoplasmic pulling influencing the centrosome's movement, which may occur even when the astral MTs have not yet reached the cortex $[14,58,59]$. This pulling is possibly mediated by cytoplasmic dynein motors, by either being anchored to actin fliaments or by carrying vesicles along the microtubules [9, 14]. Our model does not incorporate cytoplasmic pulling force and thus, cannot account for its impact on spindle elongation and positioning.

In our model for positioning the single centrosome during Interphase and bipolar spindle elongation during Anaphase B, we use the same set of parameters for studying two separate phenomena. We obtain stable positioning at the cell center for a single CS, while for two CSs of the bipolar spindle, we obtain faithful interpolar separation and positioning of individual poles. Even though we use the same parameters and the mechanism is largely similar for these scenarios, the single CS exploits the cortical interaction on both sides, while the individual poles in the bipolar spindle interact mainly with the proximal cortex. The microtubules emanating from the individual poles toward the cell center interact with those from the opposite pole via Kinesin-5 motors, thus becoming the IPMTs. Under practical scenarios, this is feasible as the microtubules that grow in the spindle inter-zone mostly form IPMTs and KMTs. Thereby a tiny proportion of them become the astral MTs that can reach the distal cortex. Without the latter, the results still hold good, as under practical circumstances, the number of such astral MTs is small and has no discernible effect on spindle dynamics.

An exciting feature of the model is that it can predict the spindle separation dynamics when MTs are short, or the cell is large compared to the average MT length. As seen in the inset of Fig. 6(a), the final interpolar distance achieved during bipolar spindle elongation mediated by astral MTs alone with low $L_{a v g}^{A M T}$ is relatively small compared to the usual scenario. The deficiency in pole separation dynamics is compensated by the IPMTs, which improve the extent of elongation and ensure that the final interpolar separation stays pretty similar even if shorter MTs mediated it. This provides the desired spindle elongation to facilitate proper cytokinesis.

Our model is developed in one dimension and based on a mean-field approach. Despite that, it can account for the duration of bipolar spindle elongation in C. elegans [41] where the astral MT mediated mechanism dominates. Since we have characterized a cell by its diameter alone, our model can be mapped closely to rod-shaped cells like those of $C$. elegans. Thus, despite its largely simplistic character, the model provides a robust base of results that manages to reveal some crucial insights into an otherwise complex biochemical system. However, despite numerous advantages, our model in one dimension is also limited by some other factors. We could not consider the effect of differing cell shapes. Therefore, cell shape-dependent spindle elongation and the correlation between the cellular aspect ratio and the final positioning of the spindle remain unexplored. As our model is also devoid of KMTs, the spindle elongation's relation to chromosomal segregation and the effect of bridging fibers are unaccounted for [32, 60]. Our model also doesn't account for interpolar attraction, mediated by minus end-directed motors, which can serve as another decisive limiting factor when it comes to elongation of the spindle [7, 61-63].

\section{APPENDIX I: PROPORTIONAL PULLING AND BUCKLING FORCES FROM CORTEX IS ESSENTIAL FOR CENTROSOME CENTERING WITH SHORT ASTRAL MTS}

Earlier, we reported a single CS position away from the cell center with short astral MTs and a given set of interaction parameters, as shown in Fig. 4(a). This occurred because the force balance in either half of the cell was dominated by forces arising from the cortex within the same half, and contribution from the other half was insignificant. To achieve centering, net pushing force arising from both the cortex must be comparable. One possible way of achieving this is to increase the buckling amplitude while keeping the dynein density unchanged. This would facilitate more pushing force for the same microtubule length allowing the CS to counter dyneinmediated pulling force at larger distances from the cell cortex. Another way is to decrease the dynein density on the cortex, such that for the same distance from the cortex, the CS experiences less pull toward the cortex. Based on these hypotheses, we simulated the model with large buckling amplitude and dynein density (see Fig. 8(a)) and small buckling amplitude and dynein density (see 8(b)). In both cases, we found CS could position at the cell center, suggesting proportional buckling and pulling forces from the cell cortex is essential for CS centering with short astral MTs.

\section{APPENDIX II: SYMMETRIC POSITIONING OF THE BIPOLAR SPINDLE WITH INITIALLY ASYMMETRIC POLES}

Fig. 9 shows dynamics of individual poles when placed asymmetrically at $t=0 \mathrm{~s}$ for normal and short astral MTs respectively. Asymmetric positioning of the poles refers to the initial spindle's center shifted away from the cell center. In other words, one of the poles is closer to the cortex than the other at the onset of spindle elongation. In Fig. 9(a) we show spindle elongation dynamics of two sets of poles placed asymmetrically on either side of the cell. Data shows that the pole closer to the cell cortex 
(a)

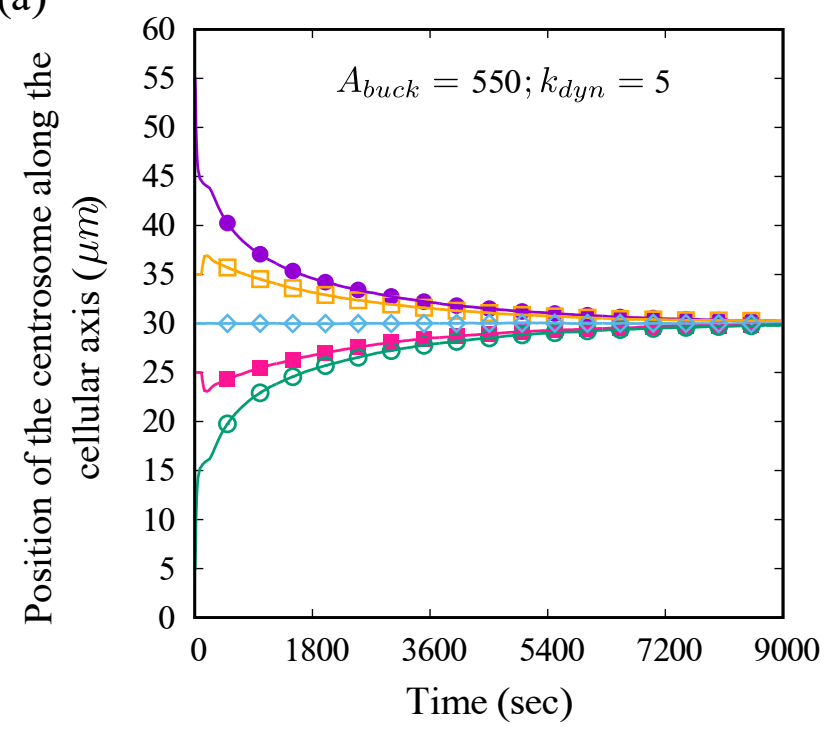

(b)

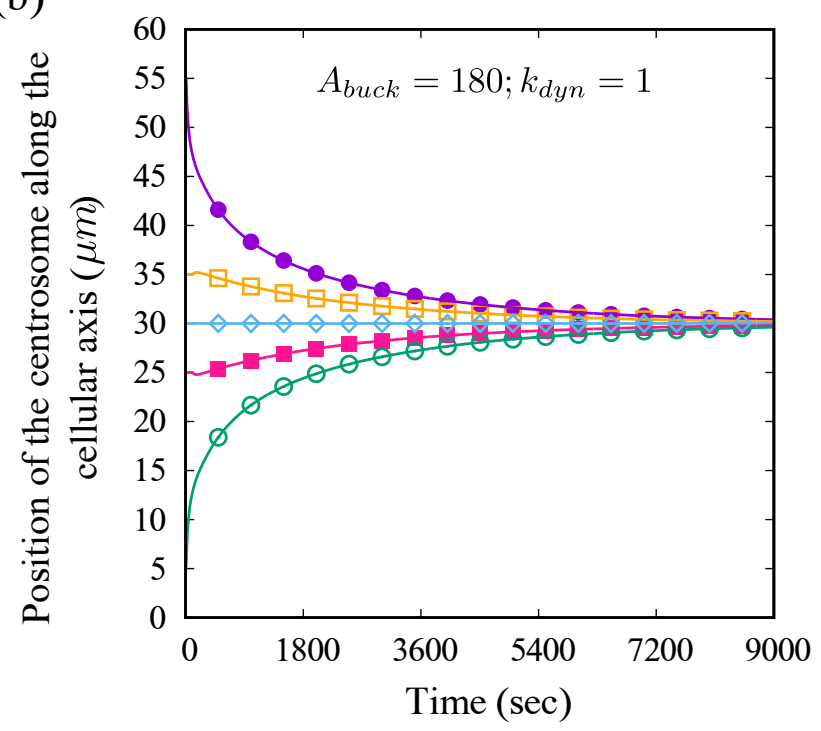

FIG. 8: Single CS positioning in a $60 \mu \mathrm{m}$ cell with short astral MTs (average length 40 um), plotted for intial CS positions at $5 \mu \mathrm{m}, 15 \mu \mathrm{m}, 25 \mu \mathrm{m}$ and $35 \mu \mathrm{m}$ away from the left cortex. (a) The mean centrosomal position for large buckling amplitude and dynein density. (b) Mean centrosomal posiiton for small buckling amplitude and dynein density.

(a)

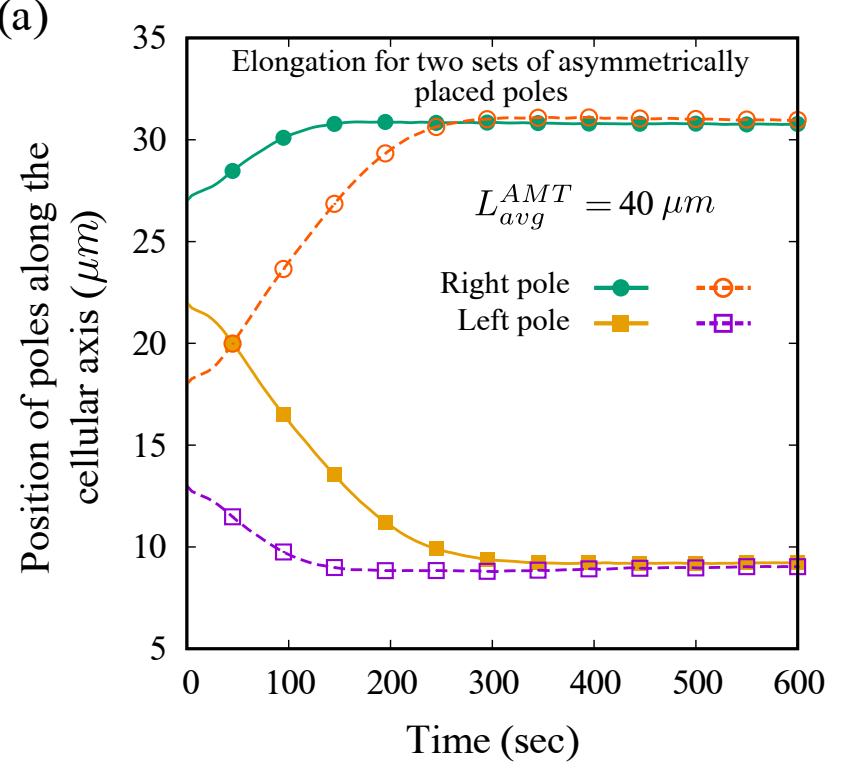

(b)

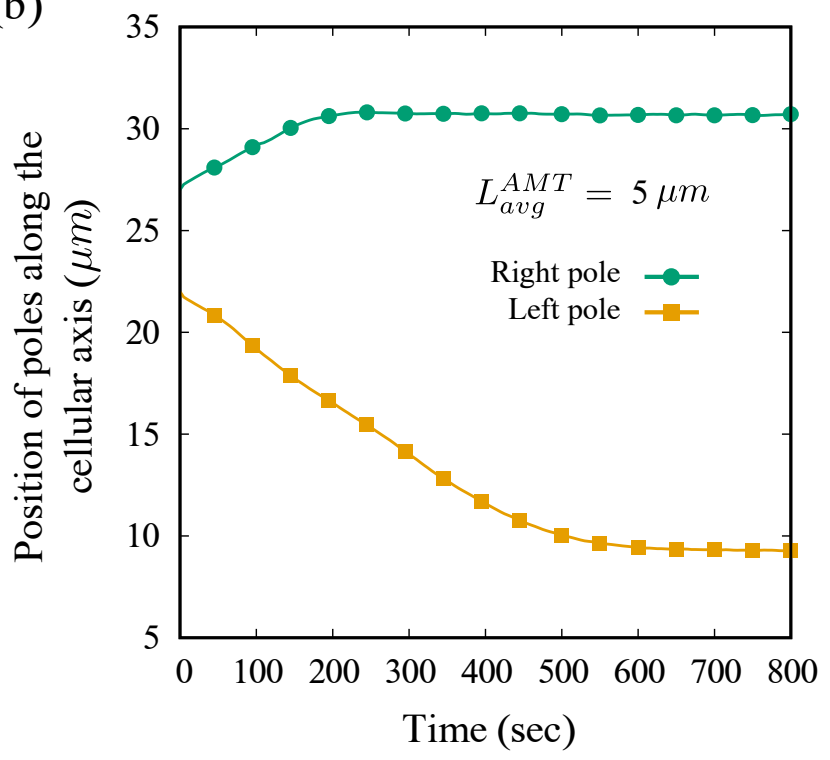

FIG. 9: Symmetric positioning of poles that were asymmetrically placed at $t=0 \mathrm{~s}$. (a) Final positioning and length of the spindle remain unaffected for varying initial positions of individual poles; (b) Pole separation kinetics for short astral MTs indicating that the symmetric positioning of the poles remain unaffected even when short astral MTs are present in the system.

moves relatively less and is positioned rapidly than the one away from the cortex. However, the final positioning of the spindle is symmetric with respect to the cell center. This occurs due to the uniform dynein density and astral MTs buckling on either side of the bipolar spindle. The spindle separation kinetics is slightly delayed with short astral MTs, as shown in Fig. 9(b). Nevertheless, the final spindle positioning and interpolar distance remain the same as observed with normal astral MTs.

\section{Acknowledgments}

We thank Alex Mogilner for his careful readings of the manuscript and fruitful suggestions for its improvement. A.M. thanks Indian Association for the Cultivation of Science (IACS), Kolkata, for support. A.S. was supported by a fellowship from the University Grants Commission (UGC), India. R.P. thanks IACS for support and Grant No. EMR/2017/001346 of SERB, DST, India for 
the computational facility.

[1] M. Kirschner and T. Mitchison, Cell 45, 329 (1986).

[2] A. Burakov, E. Nadezhdina, B. Slepchenko, and V. Rodionov, J Cell Biol 162, 963-969 (2003).

[3] I. M. Tolic-Norrelykke, Eur Biophys J. 37, 1271 (2008).

[4] N. Pavin and I. M. Tolic-Norrelykke, Syst Synth Biol. 8, 1103 (2014).

[5] N. R. Adames and J. A. Cooper, Molecular Biology of the Cell 149, 863 (2000).

[6] A. Sarkar, R. Paul, and H. Rieger, Biophysical Journal 116, 2079 (2019).

[7] S. Chatterjee, A. Sarkar, J. Zhu, A. Khodjakov, A. Mogilner, and RajaPaul, Biophysical Journal 119, 434 (2020).

[8] J. R. McIntosh, M. I. Molodtsov, and F. I. Ataullakhanov, Q Rev. Biophys. 45, 147 (2012).

[9] J. Howard and C. Garzon-Coral, BioEssays 39, 1 (2017).

[10] M. Vleugel, M. Kok, and M. Dogterom, Cell Adhesion \& Migration 10, 475 (2016).

[11] J. Zhu, A. Burakov, V. Rodionov, and A. Mogilner, Molecular Biology of the Cell 21, 4418 (2010).

[12] G. Letort, F. Nedelec, L. Blanchoin, and M. Théry, Molecular Biology of the Cell 27, 2833 (2016).

[13] L. Laan, N. Pavin, J. Husson, G. Romet-Lemonne, M. van Duijn, M. López, R. Vale, F. Jülicher, S. ReckPeterson, and M. Dogterom, Cell 148, 502 (2012).

[14] K. Kimura and A. Kimura, BioArchitecture 1, 74-79 (2011).

[15] I. V. Maly, Commun Integr Biol 4, 230-235 (2011).

[16] F. J. McNally, J Cell Biol 200, 131 (2013).

[17] S. W. Grill and A. A. Hyman, Developmental Cell 8, 461 (2005).

[18] A. M. Saunders, J. Powers, S. Strome, and W. M. Saxton, Current Biology 17 (2007).

[19] S. W. Grill, J. Howard, E. Schaffer, E. H. K. Stelzer, and A. A. Hyman, Science 301, 518 (2003).

[20] T. Nguyen-Ngoc, K. Afshar, and P. Gonczy, Nat Cell Biol. 9, 1294 (2007).

[21] C. Kozlowski, M. Srayko, and F. Nedelec, Cell 129, 499 (2007).

[22] M. Dogterom and B. Yurke, Science 278, 856 (1997).

[23] P. T. Tran, L. Marsh, V. Doye, S. Inoué, and F. Chang, J Cell Biol 153, 397 (2001).

[24] D. R. Drummond and R. A. Cross, Curr Biol 10, 766 (2000).

[25] J. Fu, I. M. Hagan, and D. M. Glover, Cold Spring Harb Perspect Biol. 7 (2015).

[26] T. Stearns, Cell 105, 417 (2001).

[27] S. L. Prosser and L. Pelletier, Nature Reviews Molecular Cell Biology 18, 187 (2017).

[28] A. Mogilner, R. Wollman, G. Civelekoglu-Scholey, and J. Scholey, Trends in Cell Biology 16, 88 (2006).

[29] Y. Li, W. Yu, Y. Liang, and X. Zhu, Cell Research 17, 701-712 (2007).

[30] H. Maiato, A. M. Gomes, F. Sousa, and M. Barisic, biology 6, 13 (2016).

[31] I. Brust-Mascher and J. M. Scholey, PubMed 39, 1149 (2011).

[32] K. Vukusic, R. Buda, and I. M. Tolic, J Cell Sci 132, jcs231985 (2019)

[33] J.-C. Labbe, E. K. McCarthy, and B. Goldstein, J. Cell Biol. 167, 245 (2004).

[34] C. Garzon-Coral, H. A. Fantana, and J. Howard, Science 352, 1124-1127 (2016).

[35] I. Brust-Mascher, G. Civelekoglu-Scholey, M. Kwon, A. Mogilner, and J. M. Scholey, PNAS 101, 15938 (2004).

[36] A. F. Straight, J. W. Sedat, and A. W. Murray, J Cell Biol. 143, 687-694 (1998).

[37] C. J. Hogan, H. Wein, L. Wordeman, J. M. Scholey, K. E. Sawin, and W. Z. Cande, PNAS 90, 6611-6615 (1993).

[38] I. Brust-Mascher and J. M. Scholey, Mol Biol Cell. 13, 3967-3975 (2002).

[39] J. R. Aist, H. Liang, and M. W. Berns, J. Cell Sci. 104, 1207 (1993).

[40] G. Fink, I. Schuchardt, J. Colombelli, E. Stelzer, and G. Steinberg, The EMBO Journal 25, 4897 (2006).

[41] Y. Hara and A. Kimura, Current Biology 19, 1549 (2009).

[42] T. Mitchison and M. Kirschner, Nature 312, 237 (1984).

[43] M. Dogterom and S. Leibler, Phys Rev Lett. 70, 1347 (1993).

[44] F. Verde, M. Dogterom, E. Stelzer, E. Karsenti, and S. Leibler, J. Cell Biol. 118, 1097 (1992).

[45] S. Som, S. Chatterjee, and R. Paul, PHYSICAL REVIEW E 99, 1 (2019).

[46] S. Sutradhar, V. Yadav, S. Sridhar, L. Sreekumar, D. Bhattacharyya, S. K. Ghosh, R. Paul, and K. Sanyal, Molecular Biology of the Cell 26, 3954 (2015).

[47] D. N. Mastronarde, K. L. McDonald, R. Ding, and J. R. McIntosh, J Cell Biol 123, 1475 (1993).

[48] E. N. Cytrynbaum, J. M. Scholey, and A. Mogilner, Biophys J. 84, 757-769 (2003).

[49] M. J. Schnitzer, K. Visscher, and S. M. Block, Nature Cell Biology 2, 718-723 (2000).

[50] D. Cole, W. Saxton, K. Sheehan, and J. Scholey, J Cell Biol 269, P22913 (1994).

[51] D. J. Sharp, K. L. McDonald, H. M. Brown, H. J. Matthies, C. Walczak, R. D. Vale, T. J. Mitchison, and J. M. Scholey, J Cell Biol 144, 125-138 (1999).

[52] R. Mallik, B. C. Carter, S. A. Lex, S. J. King, and S. P. Gross, Nature 427, 649 (2004).

[53] G. Civelekoglu-Scholey, D. J. Sharp, A. Mogilner, and J. M. Scholey, Biophys J. 90, 3966-3982 (2006).

[54] N. M. Rusan, U. S. Tulu, C. Fagerstrom, and P. Wadsworth, J Cell Biol 158, 997-1003 (2002).

[55] A. J. Zwetsloot, G. Tut, and A. Straube, Essays Biochem. 62, 725 (2018).

[56] W. H. Press, S. A. Teukolsky, W. T. Vetterling, and B. P. Flannery, Numerical Recipes in FORTRAN (2nd Ed.): The Art of Scientific Computing (Cambridge University Press, USA, 1992), ISBN 052143064X.

[57] D. Ghanti, R. W. Friddle, and D. Chowdhury, Physical Review E 98 (2018).

[58] M. S. Hamaguchi and Y. Hiramoto, Development 28 (1986).

[59] M. Wuhr, E. S. Tan, S. K. Parker, H. W. Detrich, and T. J. Mitchison, Curr Biol. 20, 2040-2045 (2010). 
[60] K. Vukusic and I. M. Tolic, Seminars in Cell \& Developmental Biology 117, 127-139 (2021).

[61] R. Basto, K. Brunk, T. Vinadogrova, N. Peel, A. Franz, A. Khodjakov, and J. W. Raff, Cell 133, 1032 (2008).

[62] M. Kwon, S. A. Godinho, N. S. Chandhok, N. J. Ganem,
A. Azioune, M. Thery, and D. Pellman, Genes Dev. 22, 2189 (2008).

[63] N. P.Ferenz, R. Paul, C. Fagerstrom, A. Mogilner, and P. Wadsworth, Current Biology 19, 1833 (2009). 